\title{
Softmax Function Based Neutrosophic Aggregation Operators and Application in Multi-Attribute Decision Making Problem
}

Totan Garai ( $\sim$ garaitotan@gmail.com )

Silda Chandra Sekhar College

Shyamal Dalapati

Bengal Engineering College: Indian Institute of Engineering Science and Technology

Florentin Smarandache

University Institute of Mexico: Instituto Universitario de Mexico

\section{Research Article}

Keywords: Softmax function, Single valued neutrosophic set, Aggregation operator, Multi attribute decision making strategy

Posted Date: July 1st, 2021

DOI: https://doi.org/10.21203/rs.3.rs-631282/v1

License: (c) (i) This work is licensed under a Creative Commons Attribution 4.0 International License. Read Full License 
Noname manuscript No.

(will be inserted by the editor)

\title{
Softmax function based neutrosophic aggregation operators and application in multi-attribute decision making problem
}

\author{
Totan Garai • Shyamal Dalapati • Florentin Smarandache \\ Received: date / Accepted: date
}

\begin{abstract}
Softmax function is a well-known generalization of the logistic function. It has been extensively used in various probabilistic classification methods such as softmax regression, linear discriminant analysis, naive bayes classifiers, and artificial neural networks. Inspired by the advantages of softmax function, we focused on this paper is to develop the softmax function based single valued neutrosophic aggregation operators. Then we have established some essential properties of aggregation operators based on softmax function with neutrosophic set. Additionally, we have defined a multi-attribute decision making method based on the proposed aggregation operators. Using this MADM method we exercised a realistic MADM problem with neutrosophic informations. Finally, we checked the validity and reliability of the proposed methods considering by one numerical illustration.
\end{abstract}

Keywords Softmax function; Single valued neutrosophic set, Aggregation operator, Multi attribute decision making strategy

\section{Introduction}

Intuitionistic fuzzy set[3] is a effectual generalization of fuzzy set [2]. But single valued neutrosophic (SVN) set [1] is a successful generalization of the fuzzy set [2]. SVN set each element is expressed by triplet of membership degrees which are membership, indeterminacy and falsity degrees. Sum of the membership degrees value lies between 0 and 3. Day by day SVNS has received more intentness by the researchers due to its formation of structure. In the decision of some modern science real problems depend on multi-attribute decision making (MADM). Because, MADM provide the best choice option to select the alternatives with respect to the attributes. How to expresses the attributes value in the decision making problem this is the great important factor. Some time

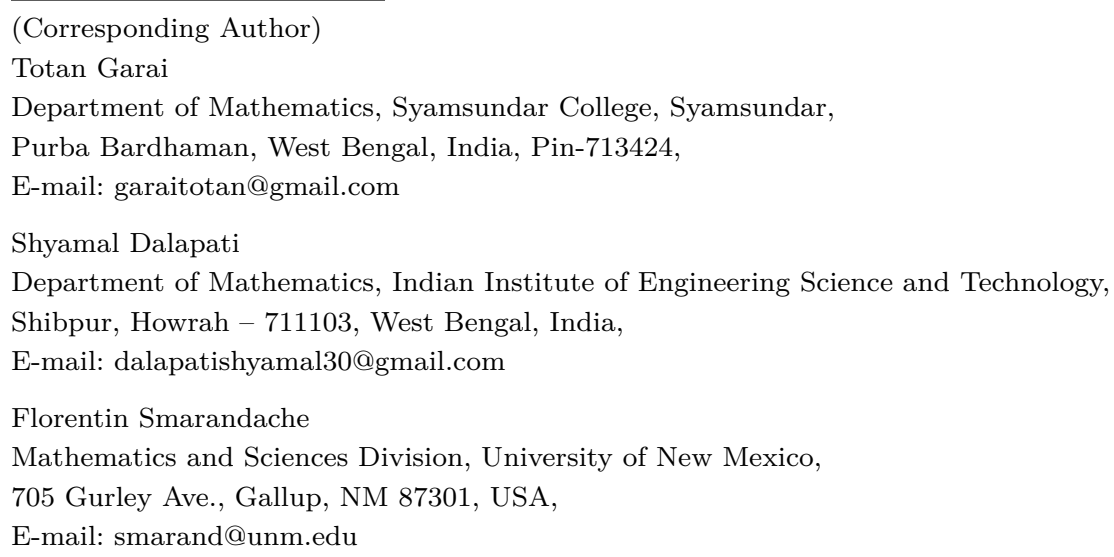


in the decision making problem, so many uncertainties and complexity occurred. In this case, SVN set has significant role to express these informations. The possibility mean variance of SVN set was developed by Garai et al. [27]. Recently many researchers proposed various strategy of their work consider by SVN set such as [4, $5,6,7,8,9]$ and so on.

In uncertain environment, some decision making (DM) problem handle by aggregation operator. Using the different aggregation operators, recently many researchers have on DM problem under SVN environment. For instances, Garg and Nancy [10] developed some new hybrid aggregation operators using arithmetic and geometric aggregation operators. They also solved one MADM problem in SVN environment. Ji et al. [11] proposed the SVNS-Frank normalized bonferroni mean (SVNFNPBM) operator to aggregate all values. This SVNFNPBM operator applied to chosen the third-party logistics example. Some arithmetic operation of SVN numbers using frank norm operators was defined by Nancy and Garg [12]. Also its applied to MADM problems.Sodenkamp et al. [5] presents an aggregation strategy for multi attribute group decision making (MAGDM) problems under SVN environment. Liu et al. [13] defined some aggregation operator by combining hamacher operations and also generalized aggregation operators in SVN environment. Recently, Chen and Ye [14] considered two operator which are Dombi weighted geometric average and Dombi weighted arithmetic average operators under SVN environment.

Liu et al. [28] developed a new single-valued neutrosophic schweizer-sklar prioritized weighted averaging (SVNSSPRWA) operator. Thereafter, he studied some basic properties of this proposed aggregation operators. Also gave the two decision making models for showing the effectiveness of this novel operators. Further, Lui et al.[29] developed the novel weighted single-valued neutrosophic power dual muirhead mean (WSVNPDMM) operator and single-valued neutrosophic power dual muirhead mean (SVNPDMM) operator. Further, based on these aggregation operators, they proposed a new technique to MAGDM problem. Tan and Zang [30] defined a new distance measure, similarity measure, and neutrosophic entropy for refined SVN sets. Rong et al. [31] defined several new operational laws of SVN number depend on Archimedean copula and co-copula (ACC) and discuss their related properties. They proposed some novel power aggregation operators (AOs) to merge SVN information, i.e., SVN copula power geometric (SVNCPG), weighted SVNCPA (WSVNCPA) operator, etc. Also, he has proposed MADM problems with SVN information usin these operators.

Now a day's, many researchers are develop some operators in SVN environment. Based on dombi t-norm and t-conorm, Chen and Ye [14] developed SVNDWGAA operator to deal with the aggregation of SVN numbers in MADM process. Li et al. [15] improved a generalized weighted geometric heronian mean (IGWGHM) operator. Also Li et al. [15] proposed the improve weighted heronian mean (NNIGWHM) operator and improve generalized weighted geometric heronian mean (NNIGWGHM) operator for neutrosophic numbers. And these operators applied to MADM problems. Garai et al. [26] proposed the new ranking of SVN-number and applied to MADM problem. Recently, Wei and Wei [6] presents some SVN-dombi prioritized average (SVNDPA) operator and SVN-Dombi prioritized geometric (SVNDPG) operator. They utilized these operators to solve MADM problems under SVNS environment.

\subsection{Motivation}

So, above discussion says that many aggregation operators are extended with the different single valued neutrosophic information. Then some researchers are successfully applied to many MADM problem and multi-atribute group decision making (MAGDM) problems under SVNS environments. But in this weighted aggregation operators have certain restriction, because most of aggregation are not applicable in the absent of SVNNs. Hence some operators cannot be applicable in some real life problems. These type of restriction handle by the softmax function. 
Previously, many researchers work on MADM under fuzzy environment using different usual aggregation operators. Torres et al. [16] firstly applied softmax function in decision making problems under hesitant fuzzy set environment. He propose a series of aggregation operator based on softmax function. Later, Yu [17] extend softmax function based aggregation operator in intuitionistic fuzzy set environment. He developed the series of aggregation operators and applied these to a MADM problem. When we ranked the different alternatives of real MADM problem under SVN environment, some have difficulties raised that time. We cannot rank the alternatives of MADM problem using normal ranking method like fuzzy number. Now, how can we rank the alternatives with single valued neutrosophic information? Also, how we can apply the softmax function based aggregation operator in MADM? What is the usefulness of softmax function based aggregation operator in MADM problem? When we studied some article relate to this research that time few question arise our mind. Therefore from that place, we try to established a best ranking method with the help of softmax function based aggregation operator.

\subsection{Novelty}

In this paper, we extend the softmax function based intuitionistic fuzzy (IF) aggregation operators to softmax function based SVN aggregation operators. Additionally, some softmax function based aggregation operators are developed here, which are softmax SVN weighted average (SVNWA) operator, Softmax SVN weighted geometric (SVNWG) operator, generalized softmax single valued neutrosophic weighted average (GSSVNWA) operator and generalized softmax SVN weighted geometric (GSSVNIFWG) operator. Then, we proposed the essential properties of the proposed softmax based aggregation operators. Further this decision making technique applied to real MADM problem.

The main contributions of the paper is that:

- We extend the SIFWA operator to SSVNWA operator.

- We extend the SIFWG operator to SSVNWG operator.

- We extend the GSIFWA operator to GSSVNWA operator.

- We extend the GSIFWG operator to GSSVNWG operator.

- We develop a MADM strategy based on the proposed operators.

- To check the validity of MADM strategy we solved one real MADM problem.

\subsection{Structure of the paper}

The rest of the paper is classified as follows: In Section 2, some basic concepts and definition related to NS, SVNS are discussed and also presented score function and accuracy function of SVNNs. In Section 3, SVNWA, SVNWG, GSSVNWA, GSSVNWG are defined and introduced its associate some basic properties and some examples. Section 4, presented a MADM strategy based on the proposed aggregation operators. In section 5, we solved a numerical example to check the validity and applicability of proposed strategy. Finally, conclusions and future research direction of this study are presented in Section 6 .

\section{Basic Preliminaries}

Definition 2.1 Let $X$ be a universe set. A nutrosophic [25] sates $\tilde{E}$ over $X$ is defined by $\tilde{E}=\left\{\left\langle x,\left(T_{\tilde{E}}(x), I_{\tilde{E}}(x)\right.\right.\right.$, $\left.\left.\left.F_{\tilde{E}}(x)\right)\right\rangle: x \in X\right\}$, where $T_{\tilde{E}}(x), I_{\tilde{E}}(x)$ and $F_{\tilde{E}}(x)$ are called truth membership function, indeterminacymembership function and falsity membership functions respectively. They are defined as

$$
\left.T_{\tilde{E}}: X \rightarrow\right]^{-} 0,1^{+}\left[, \quad I_{\tilde{E}}: X \rightarrow\right]^{-} 0,1^{+}\left[, \quad F_{\tilde{E}}: X \rightarrow\right]^{-} 0,1^{+}[
$$


such that $0^{-} \leq T_{\tilde{E}}(x)+I_{\tilde{E}}(x)+F_{\tilde{E}}(x) \leq 3^{+}$

Definition 2.2 Let $X$ be a universe set[1]. A SVN-set $\tilde{E}$ over $X$ is a neutroophic set, but the truth-membership function, indeterminacy-membership function and falsity-membership function are respectively defined by

$$
T_{\tilde{E}}: X \rightarrow[0,1], \quad I_{\tilde{E}}: X \rightarrow[0,1], \quad F_{\tilde{E}}: X \rightarrow[0,1]
$$

such that $0 \leq T_{\tilde{E}}(x)+I_{\tilde{E}}(x)+F_{\tilde{E}}(x) \leq 3$. For convenience, a SVNN can be expressed to be $\tilde{E}=\left(T_{\tilde{E}}, I_{\tilde{E}}, F_{\tilde{E}}\right), T_{\tilde{E}} \in$ $[0,1], I_{\tilde{E}} \in[0,1], F_{\tilde{E}} \in[0,1]$ and $0 \leq T_{\tilde{E}}+I_{\tilde{E}}+F_{\tilde{E}} \leq 3$.

Definition 2.3 Let $\tilde{C}=\left\{\left\langle x,\left(T_{\tilde{C}}(x), I_{\tilde{C}}(x), F_{\tilde{C}}(x)\right)\right\rangle: x \in X\right\}$ and $\tilde{E}=\left\{\left\langle x,\left(T_{\tilde{E}}(x), I_{\tilde{E}}(x), F_{\tilde{E}}(x)\right)\right\rangle: x \in X\right\}$ be two SVN-sets in $X$, then operations between them defined [1] as follows:

(i) $\tilde{C} \subseteq \tilde{E}$ iff $T_{\tilde{C}}(x) \leq T_{\tilde{E}}(x), I_{\tilde{C}}(x) \geq I_{\tilde{E}}(x), T_{\tilde{C}}(x) \geq T_{\tilde{E}}(x)$ for all $x \in X$.

(ii) $\tilde{C}=\tilde{E}$ iff $\tilde{C} \subseteq \tilde{E}$ and $\tilde{E} \subseteq \tilde{C}$ for all $x \in X$.

(iii) $\tilde{E}^{c}=\left\{\left\langle x,\left(F_{\tilde{E}}(x), 1-I_{\tilde{E}}(x), T_{\tilde{E}}(x)\right)\right\rangle: x \in X\right\}$ for all $x \in X$.

(iv) $\tilde{C} \cup \tilde{E}=\left\{\left\langle x, \max \left(T_{\tilde{C}}(x), T_{\tilde{E}}(x)\right), \min \left(I_{\tilde{C}}(x), I_{\tilde{E}}(x)\right), \min \left(F_{\tilde{C}}(x), F_{\tilde{E}}(x)\right)\right\rangle: x \in X\right\}$ for all $x \in X$.

(v) $\tilde{C} \cap \tilde{E}=\left\{\left\langle x, \min \left(T_{\tilde{C}}(x), T_{\tilde{E}}(x)\right), \max \left(I_{\tilde{C}}(x), I_{\tilde{E}}(x)\right), \max \left(F_{\tilde{C}}(x), F_{\tilde{E}}(x)\right)\right\rangle: x \in X\right\}$ for all $x \in X$.

Definition 2.4 Let $\tilde{E}, \tilde{E}_{1}, \tilde{E}_{2}$ be three SVN-sets in $X$. Then, the arithmetic [1] operations are defined as follows:

(i) $\tilde{E}_{1}+\tilde{E}_{2}=\left\{\left\langle x, T_{\tilde{E}_{1}}(x)+T_{\tilde{E}_{2}}(x)-T_{\tilde{E}_{1}}(x) \cdot T_{\tilde{E}_{2}}(x), I_{\tilde{E}_{1}}(x) \cdot I_{\tilde{E}_{2}}(x), F_{\tilde{E}_{1}}(x) \cdot F_{\tilde{E}_{2}}(x)\right\rangle: x \in X\right\}$ for all $x \in X$.

(ii) $\tilde{E}_{1} \cdot \tilde{E}_{2}=\left\{\left\langle x, T_{\tilde{E}_{1}}(x) \cdot T_{\tilde{E}_{2}}(x), I_{\tilde{E}_{1}}(x)+I_{\tilde{E}_{2}}(x)-I_{\tilde{E}_{1}}(x) \cdot I_{\tilde{E}_{2}}(x), F_{\tilde{E}_{1}}(x)+F_{\tilde{E}_{2}}(x)-F_{\tilde{E}_{1}}(x) \cdot F_{\tilde{E}_{2}}(x)\right\rangle: x \in X\right\}$ for all $x \in X$.

(iii) $\quad \lambda . \tilde{E}=\left\{\left\langle x,\left(1-\left(1-T_{\tilde{E}}(x)\right)^{\lambda},\left(I_{\tilde{E}}(x)\right)^{\lambda},\left(F_{\tilde{E}}(x)\right)^{\lambda}\right\rangle: x \in X\right\}\right.$ for all $x \in X$.

(iv) $\tilde{E}^{\lambda}=\left\{\left\langle x,\left(T_{\tilde{E}}(x)\right)^{\lambda}, 1-\left(1-I_{\tilde{E}}(x)\right)^{\lambda}, 1-\left(1-F_{\tilde{E}}(x)\right)^{\lambda}\right\rangle: x \in X\right\}$ for all $x \in X$, Where $\lambda>0$ is a parameter.

Definition 2.5 For any SVN set, the ranking method is very significant and many research results have been received (Zhang et al. [18], Wang et al. [1]). Zhang et al. [18] given a method based on score function and accuracy function. For any SVN-set $A=\left(T_{A}, I_{A}, F_{A}\right)$, the accuracy and score function defined as:

The score function of $\tilde{E}$ is

$$
S(\tilde{E})=\frac{2+T_{\tilde{E}}-I_{\tilde{E}}-F_{\tilde{E}}}{3}, S(\tilde{E}) \in[0,1]
$$

and the accuracy function of $\tilde{E}$ is

$$
H(\tilde{E})=T_{\tilde{E}}-F_{\tilde{E}}, H(\tilde{E}) \in[-1,1]
$$

Zhang et al.[18] gave an order relation between two SVN numbers, which is defined as follows:

Definition 2.6 Let $\tilde{C}=\left(T_{\tilde{C}}, I_{\tilde{C}}, F_{\tilde{C}}\right)$ and $\tilde{E}=\left(T_{\tilde{E}}, I_{\tilde{E}}, F_{\tilde{E}}\right)$ be two SVNNs.

Now, if $S(A)>S(B)$, then $\tilde{C} \succ \tilde{E}$. Again if $S(\tilde{C})=S(\tilde{E})$, then

(i) If $H(\tilde{C})=H(\tilde{C})$, then $\tilde{C} \approx \tilde{E}$.

(ii) If $H(\tilde{C})>H(\tilde{E})$, then $\tilde{C} \succ \tilde{E}$.

\section{Softmax function based aggregation operators}

In this section we have discuss about softmax function and its important properties. Here we established some rigorous method related to softmax function based aggregation operator. 


\subsection{Softmax function}

Softmax function is a generalization form of the logistic function in the area of mathematics. It has been progressively applied to many fields of research, for instance, machine learning $[19,20]$ and decision making $[16$, 17]. The mathematical form of softmax function represented as follows:

$$
\phi_{k}\left(j, \vartheta_{1}, \vartheta_{2}, \ldots, \vartheta_{n}\right)=\phi_{k}^{j}=\frac{\exp \left(\vartheta_{j} / k\right)}{\sum_{j=1}^{n} \exp \left(\vartheta_{j} / k\right)}, k>0 .
$$

For the SVN-sets $\alpha_{j}(j=1,2,3, \ldots, n), S_{j}$ is the score value of SVN-number $\alpha_{j}$. Every $\vartheta_{j}$ is formulated by given the equation

$$
\vartheta_{j}= \begin{cases}\prod_{i=1}^{j-1} S_{i}, \mathrm{j} & =2,3, \ldots, n \\ 1 & \mathrm{j}=1\end{cases}
$$

where $k$ is the modulation parameter.

Definition 3.2 Some properties of softmax function [17] are defined as follows:

(i) $0 \leq \phi_{k}^{j} \leq 1$.

(ii) $\sum_{j=1}^{n} \phi_{k}^{j}=1$.

Softmax function [16] has the non linear characteristic, monotonicity and boundedness properties.

\subsection{SVN-sets aggregation operators based on softmax function}

In this section, we have extend the softmax function based IF aggregation operators, such as softmax IF weighted average operator (SIFWA), softmax IF weighted geometric (SIFWG) operator, generalized softmax IF weighted average (GSIFWA) operator and generalized softmax IF weighted geometric (GSIFWG) operator to softmax SVN weighted average (SVNWA) operator, softmax SVN weighted geometric (SVNWG) operator, generalized softmax SVN weighted average (GSSVNWA) operator and generalized softmax SVN weighted geometric (GSSVNIFWG) operator, respectively.

Definition 3.4 Let $\alpha_{j}(j=1,2, \ldots, n)$ be a collection of SVNNs. Then softmax single valued neutrosophic weighted average (SSVNWA) operator is a function from $\alpha^{n} \rightarrow \alpha$ such that

$$
S S V N W A\left(\alpha_{1}, \alpha_{2}, \ldots, \alpha_{n}\right)=\oplus_{j=1}^{n}\left(\phi_{k}^{j} \alpha_{j}\right)=\left(\phi_{k}^{1} \alpha_{1}\right) \oplus\left(\phi_{k}^{2} \alpha_{2}\right) \oplus \ldots \oplus\left(\phi_{k}^{n} \alpha_{n}\right)
$$

where, $\phi_{k}^{j}=\frac{\exp \left(\vartheta_{j} / k\right)}{\sum_{j=1}^{n} \exp \left(\vartheta_{j} / k\right)}, \vartheta_{j}=\left\{\begin{array}{l}\prod_{i=1}^{j-1} S_{i}, \mathrm{j}=2,3, \ldots, n \\ 1 \quad \mathrm{j}=1\end{array}, S_{i}\right.$ is the score function of the SVN-number $\alpha_{i}$.

Theorem 1 Let $\alpha_{j}(j=1,2, \ldots, n)$ be a collection of SVN-numbers, then aggregated value of SVN-numbers using the SSVNWA operation is also a SVN-number. The SSVNWA operator can be generated as:

$$
S S V N W A\left(\alpha_{1}, \alpha_{2}, \ldots, \alpha_{n}\right)=\left(1-\prod_{j=1}^{n}\left(1-T_{j}\right)^{\frac{\exp \left(\vartheta_{j} / k\right)}{\sum_{j=1}^{n} \exp \left(\vartheta_{j} / k\right)}}, \prod_{j=1}^{n}\left(I_{j}\right)^{\frac{\exp \left(\vartheta_{j} / k\right)}{\sum_{j=1}^{n} \exp \left(\vartheta_{j} / k\right)}}, \prod_{j=1}^{n}\left(F_{j}\right)^{\frac{\exp \left(\vartheta_{j} / k\right)}{\sum_{j=1}^{n} \exp \left(\vartheta_{j} / k\right)}}\right)
$$

Proof: We proof the above theorem 1 by using mathematical induction. For $n=1$, we have:

$$
\operatorname{SSVNWA}\left(\alpha_{1}, \alpha_{2}, \ldots, \alpha_{n}\right)=\left(1-\prod_{j=1}^{1}\left(1-T_{j}\right), \prod_{j=1}^{1}\left(I_{j}\right), \prod_{j=1}^{1}\left(F_{j}\right)\right)=\left(T_{1}, I_{1}, F_{1}\right) .
$$


Since for $n=1, \frac{\exp \left(\vartheta_{j} / k\right)}{\sum_{j=1}^{n} \exp \left(\vartheta_{j} / k\right)}=1$. Thus Eq.(5) holds for $\mathrm{n}=1$. Assume that the Eq. (5) holds for $\mathrm{n}=\mathrm{m}$, $S S V N W A\left(\alpha_{1}, \alpha_{2}, \ldots, \alpha_{m}\right)=\left(1-\prod_{j=1}^{m}\left(1-T_{j}\right)^{\frac{\exp \left(\vartheta_{j} / k\right)}{\sum_{j=1}^{m} \exp \left(\vartheta_{j} / k\right)}}, \prod_{j=1}^{m}\left(I_{j}\right)^{\frac{\exp \left(\vartheta_{j} / k\right)}{\sum_{j=1}^{m}\left(\vartheta^{\exp \left(\vartheta_{j} / k\right)}\right.}}, \prod_{j=1}^{m}\left(F_{j}\right)^{\frac{\exp \left(\vartheta_{j} / k\right)}{\sum_{j=1}^{m} \exp \left(\vartheta_{j} / k\right)}}\right)$

Now we prove that the Eq. (5) holds for $n=m+1$.

$$
\begin{aligned}
& S S V N W A\left(\alpha_{1}, \alpha_{2}, \ldots, \alpha_{m}, \alpha_{m+1}\right)=\left(\phi_{k}^{1} \alpha_{1}\right) \oplus\left(\phi_{k}^{2} \alpha_{2}\right) \ldots \oplus\left(\phi_{k}^{m} \alpha_{m}\right) \oplus\left(\phi_{k}^{m+1} \alpha_{m+1}\right) \\
& =\left(\left(1-\prod_{j=1}^{m}\left(1-T_{j}\right)^{\frac{\exp \left(\vartheta_{j} / k\right)}{\sum_{j=1}^{m} \exp \left(\vartheta_{j} / k\right)}}\right)+\left(1-\left(1-T_{m+1}\right)^{\frac{\exp \left(\vartheta_{m}+1 / k\right)}{\sum_{j=1}^{m+1} \exp \left(\vartheta_{j} / k\right)}}\right)\right. \\
& -\left(\left(1-\prod_{j=1}^{m}\left(1-T_{j}\right)^{\frac{\exp \left(\vartheta_{j} / k\right)}{\sum_{j=1}^{m} \exp \left(\vartheta_{j} / k\right)}}\right) \times\left(1-\left(1-T_{m+1}\right)^{\frac{\exp \left(\vartheta_{m+1} / k\right)}{\sum_{j=1}^{m+1} \exp \left(\vartheta_{j} / k\right)}}\right)\right), \\
& \left.\prod_{j=1}^{m+1}\left(I_{j}\right)^{\frac{\exp \left(\vartheta_{j} / k\right)}{\sum_{j=1}^{m+1} \exp \left(\vartheta_{j} / k\right)}}, \prod_{j=1}^{m+1}\left(F_{j}\right)^{\frac{\exp \left(\vartheta_{j} / k\right)}{\sum_{j=1}^{m+1} \exp \left(\vartheta_{j} / k\right)}}\right) \\
& =\left(1-\prod_{j=1}^{m+1}\left(1-T_{j}\right)^{\frac{\exp \left(\vartheta_{j} / k\right)}{\sum_{j=1}^{m+1} \exp \left(\vartheta_{j} / k\right)}}, \prod_{j=1}^{m+1}\left(I_{j}\right)^{\frac{\exp \left(\vartheta_{j} / k\right)}{\sum_{j=1}^{m+1} \exp \left(\vartheta_{j} / k\right)}}, \prod_{j=1}^{m+1}\left(F_{j}\right)^{\frac{\exp \left(\vartheta_{j} / k\right)}{\sum_{j=1}^{m+1} \exp \left(\vartheta_{j} / k\right)}}\right)
\end{aligned}
$$

Therefore, Eq. (5) holds for $n=m+1$, hence the Eq. (5) holds for all positive integer by principle of mathematical induction. Hence, the proof of the theorem is completed.

Example 1 Let $\alpha_{1}=(0.6,0.4,0.5), \alpha_{2}=(0.7,0.3,0.5), \alpha_{3}=(0.8,0.3,0.4)$ and $\alpha_{4}=(0.7,0.4,0.5)$ be the four SVN-numbers. Rank the four SVN-numbers using SSVNWA operator.

Solution: Here we have used the SSVNWA operator to aggregate the four SVN-numbers.

At first, we calculated the score values of four SVN-numbers using Eq. (1).

$S\left(\alpha_{1}\right)=0.567, S\left(\alpha_{2}\right)=0.633, S\left(\alpha_{3}\right)=0.700, S\left(\alpha_{4}\right)=0.600$

$\vartheta_{1}=1, \vartheta_{2}=0.567, \vartheta_{3}=0.359, \vartheta_{4}=0.251$.

To calculate $\exp \left(\vartheta_{j} / k\right)$ we take $\mathrm{k}=1$, then $\exp \left(\vartheta_{1} / k\right)=2.718, \exp \left(\vartheta_{2} / k\right)=1.763, \exp \left(\vartheta_{3} / k\right)=1.432, \exp \left(\vartheta_{4} / k\right)=$ 1.285

$$
\begin{aligned}
& \text { and } \frac{\exp \left(\vartheta_{1} / k\right)}{\sum_{j=1}^{4} \exp \left(\vartheta_{j} / k\right)}=0.378, \frac{\exp \left(\vartheta_{2} / k\right)}{\sum_{j=1}^{4} \exp \left(\vartheta_{j} / k\right)}=0.245, \frac{\exp \left(\vartheta_{3} / k\right)}{\sum_{j=1}^{4} \exp \left(\vartheta_{j} / k\right)}=0.199, \frac{\exp \left(\vartheta_{4} / k\right)}{\sum_{j=1}^{4} \exp \left(\vartheta_{j} / k\right)}=0.178 \\
& S S V N W A\left(\alpha_{1}, \alpha_{2}, \alpha_{3}, \alpha_{4}\right)=\left(\left(1-(1-0.6)^{0.378} \times(1-0.7)^{0.245} \times(1-0.8)^{0.199} \times(1-0.7)^{0.178}\right),\right. \\
& \left((0.4)^{0.378} \times(0.3)^{0.245} \times(0.3)^{0.199} \times(0.4)^{0.178}\right), \\
& \left.\left((0.5)^{0.378} \times(0.5)^{0.245} \times(0.4)^{0.199} \times(0.5)^{0.178}\right)\right) \\
& =(0.691,0.352,0.478)
\end{aligned}
$$

\subsubsection{Properties of SSVNWA operator}

\section{Property 1: Idem-potency}

If $\alpha_{1}=\alpha_{2}=\ldots=\alpha_{n}=\alpha($ say $)$, then $\operatorname{SSVNWA}\left(\alpha_{1}, \alpha_{2}, \ldots, \alpha_{n}\right)=\alpha$

Proof : Let $\alpha_{j}=\left\langle T_{j}, I_{j}, F_{j}\right\rangle,(j=1,2,3 \ldots, n)$ and $\alpha=\langle T, I, F\rangle$.

Since all $\alpha_{j}$ are equal based on Theorem (1), we get

$S S V N W A(\alpha, \alpha, \ldots, \alpha)=\left(1-(1-T)^{\sum_{j=1}^{n} \phi_{k}^{j}},(I)^{\sum_{j=1}^{n} \phi_{k}^{j}},(F)^{\sum_{j=1}^{n} \phi_{k}^{j}}\right)=\langle T, I, F\rangle=\alpha$.

Since, $\phi_{k}^{j}=\frac{\exp \left(\vartheta_{j} / k\right)}{\sum_{j=1}^{n} \exp \left(\vartheta_{j} / k\right)}, k>0$ and $\sum_{j=1}^{n} \phi_{k}^{j}=1$. 


\section{Property 2: Monotonicity}

Let $\alpha_{j}(j=1,2, \ldots, n)$ and $\beta_{j}(j=1,2, \ldots, n)$ be any two sets of SVN-numbers. If $\alpha_{j} \leq \beta_{j}$ for any $j$, then $S S V N W A\left(\alpha_{1}, \alpha_{2}, \ldots, \alpha_{n}\right) \leq S S V N W A\left(\beta_{1}, \beta_{2}, \ldots, \beta_{n}\right)$.

Proof Based on the Theorem (1), we get

$S S V N W A\left(\alpha_{1}, \alpha_{2}, \ldots, \alpha_{n}\right)=\left(1-\prod_{j=1}^{n}\left(1-T_{\alpha_{j}}\right)^{\frac{\exp \left(\vartheta_{\alpha_{j}} / k\right)}{\sum_{j=1}^{n} \exp \left(\vartheta_{\alpha_{j}} / k\right)}}, \prod_{j=1}^{n}\left(I_{\alpha_{j}}\right)^{\frac{\exp \left(\vartheta_{\alpha_{j}} / k\right)}{\sum_{j=1}^{n} \exp \left(\vartheta_{\alpha_{j}} / k\right)}}, \prod_{j=1}^{n}\left(F_{\alpha_{j}}\right)^{\frac{\exp \left(\vartheta \alpha_{j} / k\right)}{\sum_{j=1}^{n} \exp \left(\vartheta \vartheta_{j} / k\right)}}\right)$ and

$S S V N W A\left(\beta_{1}, \beta_{2}, \ldots, \beta_{n}\right)=\left(1-\prod_{j=1}^{n}\left(1-T_{\beta_{j}}\right)^{\frac{\exp \left(\vartheta_{\beta_{j}} / k\right)}{\sum_{j=1}^{n} \exp \left(\vartheta_{\beta_{j}} / k\right)}}, \prod_{j=1}^{n}\left(I_{\beta_{j}}\right)^{\frac{\exp \left(\vartheta_{\beta_{j}} / k\right)}{\sum_{j=1}^{n} \exp \left(\vartheta_{\beta_{j}} / k\right)}}, \prod_{j=1}^{n}\left(F_{\beta_{j}}\right)^{\frac{\exp \left(\vartheta_{\beta_{j}} / k\right)}{\sum_{j=1}^{n} \exp \left(\vartheta_{\beta_{j}} / k\right)}}\right)$

Since all $\alpha_{j} \leq \beta_{j}(j=1,2, \ldots, n)$. Therefore,

$$
\begin{aligned}
T_{\alpha_{j}} \leq T_{\beta_{j}} & \Rightarrow\left(1-T_{\alpha_{j}}\right) \geq\left(1-T_{\beta_{j}}\right) \\
& \Rightarrow\left(1-T_{\alpha_{j}}\right)^{\frac{\exp \left(\vartheta_{\alpha_{j}} / k\right)}{\sum_{j=1}^{n} \exp \left(\vartheta_{\alpha_{j}} / k\right)}} \geq\left(1-T_{\beta_{j}}\right)^{\frac{\exp \left(\vartheta_{\beta_{j}} / k\right)}{\sum_{j=1}^{n} \exp \left(\vartheta_{j} / k\right)}} \\
& \Rightarrow \prod_{j=1}^{n}\left(1-T_{\alpha_{j}}\right)^{\frac{\exp \left(\vartheta_{\alpha_{j}} / k\right)}{\sum_{j=1}^{n} \exp \left(\vartheta_{\alpha_{j}} / k\right)}} \geq \prod_{j=1}^{n}\left(1-T_{\beta_{j}}\right)^{\frac{\exp \left(\vartheta_{\beta_{j}} / k\right)}{\sum_{j=1}^{n} \exp \left(\vartheta_{\beta_{j}} / k\right)}} \\
& \Rightarrow\left(1-\prod_{j=1}^{n}\left(1-T_{\alpha_{j}}\right)^{\frac{\exp \left(\vartheta_{\alpha_{j}} / k\right)}{\sum_{j=1}^{n} \exp \left(\vartheta_{\alpha_{j}} / k\right)}}\right) \leq\left(1-\prod_{j=1}^{n}\left(1-T_{\beta_{j}}\right)^{\frac{\exp _{j=1}^{n}\left(\vartheta_{\beta_{j}} / k\right)}{\exp \left(\vartheta_{\beta_{j}} / k\right)}}\right)
\end{aligned}
$$

Further,

$$
\begin{aligned}
I_{\alpha_{j}} \geq I_{\beta_{j}} & \Rightarrow\left(I_{\alpha_{j}}\right)^{\frac{\exp \left(\vartheta_{\alpha_{j}} / k\right)}{\sum_{j=1}^{n} \exp \left(\vartheta_{\alpha_{j}} / k\right)}} \geq\left(I_{\beta_{j}}\right)^{\frac{\exp \left(\vartheta_{\beta_{j}} / k\right)}{\sum_{j=1}^{n} \exp \left(\vartheta_{\beta_{j}} / k\right)}} \\
& \Rightarrow \prod_{j=1}^{n}\left(I_{\alpha_{j}}\right)^{\frac{\exp \left(\vartheta_{\alpha_{j}} / k\right)}{\sum_{j=1}^{n} \exp \left(\vartheta_{\alpha_{j}} / k\right)}} \geq \prod_{j=1}^{n}\left(I_{\beta_{j}}\right)^{\sum_{j=1}^{n} \exp \left(\vartheta_{\beta_{j}} / k\right)}
\end{aligned}
$$

Similarly, we have also

$$
\prod_{j=1}^{n}\left(F_{\alpha_{j}}\right)^{\frac{\exp \left(\vartheta_{\alpha_{j}} / k\right)}{\sum_{j=1}^{n} \exp \left(\vartheta_{\alpha_{j}} / k\right)}} \geq\left(F_{\beta_{j}}\right)^{\frac{\exp \left(\vartheta_{\beta_{j}} / k\right)}{\sum_{j=1}^{n} \exp \left(\vartheta_{\beta_{j}} / k\right)}}
$$

Hence the proof is complete.

\section{Property 3: Boundedness}

Let $\alpha_{j}(j=1,2, \ldots, n)$ be any set of SVN-number. If $\alpha^{-}=\min \left\{\alpha_{j}\right\}$ and $\alpha^{+}=\max \left\{\alpha_{j}\right\}$, then $\alpha^{-} \leq$ $S S V N W A\left(\alpha_{1}, \alpha_{2}, \ldots, \alpha_{n}\right) \leq \alpha^{+}$.

Proof : Let $\alpha^{+}=\max \left\{\alpha_{1}, \alpha_{2}, \ldots, \alpha_{n}\right\}$ and $\alpha^{-}=\min \left\{\alpha_{1}, \alpha_{2}, \ldots, \alpha_{n}\right\}$. According to properties 1 and 2 , we have $S S V N W A\left(\alpha_{1}, \alpha_{2}, \ldots, \alpha_{n}\right) \geq S S V N W A\left(\alpha^{-}, \alpha^{-}, \ldots, \alpha^{-}\right)=\alpha^{-}$and $S S V N W A\left(\alpha_{1}, \alpha_{2}, \ldots, \alpha_{n}\right) \leq S S V N W A\left(\alpha^{+}, \alpha^{+}, \ldots, \alpha^{+}\right)=\alpha^{+}$ So, we have $\alpha^{-} \leq S S V N W A\left(\alpha_{1}, \alpha_{2}, \ldots, \alpha_{n}\right) \leq \alpha^{+}$.

Hence the proof is complete.

Definition 3.5 Let $\alpha_{j}(j=1,2, \ldots, n)$ be a collection of SVN-numbers. Then softmax single valued neutrosophic weighted geometric (SSVNWG) operator is a function from $\alpha^{n} \rightarrow \alpha$ such that

$$
S S V N W G\left(\alpha_{1}, \alpha_{2}, \ldots, \alpha_{n}\right)=\oplus_{j=1}^{n}\left(\phi_{k}^{j} \alpha_{j}\right)=\otimes_{j=1}^{n}\left(\alpha_{j}\right)^{\phi_{k}^{j}}=\left(\alpha_{1}\right)^{\phi_{k}^{1}} \otimes\left(\alpha_{2}\right)^{\phi_{k}^{2}} \otimes \ldots \otimes\left(\alpha_{n}\right)^{\phi_{k}^{n}}
$$


where $\phi_{k}^{j}=\frac{\exp \left(\vartheta_{j} / k\right)}{\sum_{j=1}^{n} \exp \left(\vartheta_{j} / k\right)}, \vartheta_{j}=\left\{\begin{array}{ll}\prod_{i=1}^{j-1} S_{i}, \mathrm{j}=2,3, \ldots, n \\ 1 \quad \mathrm{j}=1\end{array}\right.$ and $S_{i}$ is the score function of the SVN-number $\alpha_{i}$.

Theorem 2 Let $\alpha_{j}(j=1,2, \ldots, n)$, be a collection of SVNNs, then aggregated value of SVN-numbers using the SSVNWG operation is also a SVN-number and

$$
\begin{aligned}
& S S V N W G\left(\alpha_{1}, \alpha_{2}, \ldots, \alpha_{n}\right)=\left(\prod_{j=1}^{n}\left(T_{j}\right)^{\frac{\exp \left(\vartheta_{j} / k\right)}{\sum_{j=1}^{n} \exp \left(\vartheta_{j} / k\right)}},\right. \\
& \left.1-\prod_{j=1}^{n}\left(1-I_{j}\right)^{\frac{e x p\left(\vartheta_{j} / k\right)}{\sum_{j=1}^{n} \exp \left(\vartheta_{j} / k\right)}}, 1-\prod_{j=1}^{n}\left(1-F_{j}\right)^{\frac{\exp \left(\vartheta_{j} / k\right)}{\sum_{j=1}^{n} \exp \left(\vartheta_{j} / k\right)}}\right)
\end{aligned}
$$

Proof : We proof the above Theorem 2 by using mathematical induction.

For $n=1$, from the Eq. (7) we have

$$
\begin{aligned}
S S V N W G\left(\alpha_{1}, \alpha_{2}, \ldots, \alpha_{n}\right)= & \left(\prod_{j=1}^{1}\left(T_{j}\right)^{\frac{\exp \left(\vartheta_{j} / k\right)}{\sum_{j=1}^{n} \exp \left(\vartheta_{j} / k\right)}},\right. \\
& \left.1-\prod_{j=1}^{1}\left(1-I_{j}\right)^{\frac{\exp \left(\vartheta_{j} / k\right)}{\sum_{j=1}^{n} \exp \left(\vartheta_{j} / k\right)}}, 1-\prod_{j=1}^{1}\left(1-F_{j}\right)^{\frac{\exp \left(\vartheta_{j} / k\right)}{\sum_{j=1}^{n} \exp \left(\vartheta_{j} / k\right)}}\right)=\left\langle T_{1}, I_{1}, F_{1}\right\rangle
\end{aligned}
$$

Since, for $n=1, \frac{\exp \left(\vartheta_{j} / k\right)}{\sum_{j=1}^{n} \exp \left(\vartheta_{j} / k\right)}=1$. Thus Eq.(7) holds for $n=1$. Assume that the Eq. (7) holds for $n=m$,

$$
\begin{aligned}
S S V N W G\left(\alpha_{1}, \alpha_{2}, \ldots, \alpha_{m}\right)= & \left(\prod_{j=1}^{m}\left(T_{j}\right)^{\frac{\exp \left(\vartheta_{j} / k\right)}{\left.\sum_{j=1}^{m}=\exp _{j} / k\right)}},\right. \\
& \left.1-\prod_{j=1}^{m}\left(1-I_{j}\right)^{\frac{\exp \left(\vartheta_{j} / k\right)}{\sum_{j=1}^{m}\left(\vartheta^{m} / k\right)}}, 1-\prod_{j=1}^{m}\left(1-F_{j}\right)^{\frac{\exp \left(\vartheta_{j} / k\right)}{\sum_{j=1}^{m}\left(\vartheta^{\exp \left(\vartheta_{j} / k\right)}\right.}}\right)
\end{aligned}
$$


Now we have prove that the Eq. (7) hold for $n=m+1$. Then

$$
\begin{aligned}
& S S V N W G\left(\alpha_{1}, \alpha_{2}, \ldots, \alpha_{m}, \alpha_{m+1}\right)=\left(\alpha_{1}\right)^{\phi_{k}^{1}} \otimes\left(\alpha_{2}\right)^{\phi_{k}^{2}} \otimes \ldots \otimes\left(\alpha_{m}\right)^{\phi_{k}^{m}} \otimes\left(\alpha_{m+1}\right)^{\phi_{k}^{m+1}} \\
& =\left(\prod_{j=1}^{m}\left(T_{j}\right)^{\frac{\exp \left(\vartheta_{j} / k\right)}{\sum_{j=1}^{m} \exp \left(\vartheta_{j} / k\right)}}, 1-\prod_{j=1}^{m}\left(1-I_{j}\right)^{\frac{\exp \left(\vartheta_{j} / k\right)}{\sum_{j=1}^{m} \exp \left(\vartheta_{j} / k\right)}},\right. \\
& \left.1-\prod_{j=1}^{m}\left(1-F_{j}\right)^{\frac{\exp \left(\vartheta_{j} / k\right)}{\sum_{j=1}^{m} \exp \left(\vartheta_{j} / k\right)}}\right) \\
& =\left(\prod_{j=1}^{m}\left(T_{j}\right)^{\frac{\exp \left(\vartheta_{j} / k\right)}{\sum_{j=1}^{m} \exp \left(\vartheta_{j} / k\right)}} \times\left(T_{m+1}\right)^{\frac{\exp \left(\vartheta_{m+1} / k\right)}{\sum_{j=1}^{m+1} \exp \left(\vartheta_{j} / k\right)}},\right. \\
& \prod_{j=1}^{m}\left(I_{j}\right)^{\frac{\exp \left(\vartheta_{j} / k\right)}{\sum_{j=1}^{m} \exp \left(\vartheta_{j} / k\right)}}+\left(I_{m+1}\right)^{\frac{\exp \left(\vartheta_{m+1} / k\right)}{\sum_{j=1}^{m+1} \exp \left(\vartheta_{j} / k\right)}}-\prod_{j=1}^{m}\left(I_{j}\right)^{\frac{\exp \left(\vartheta_{j} / k\right)}{\sum_{j=1}^{m} \exp \left(\vartheta_{j} / k\right)}} \\
& \times\left(I_{m+1}\right)^{\frac{\exp \left(\vartheta_{m+1} / k\right)}{\sum_{j=1}^{m+1} \exp \left(\vartheta_{j} / k\right)}}, \prod_{j=1}^{m}\left(F_{j}\right)^{\frac{\exp \left(\vartheta_{j} / k\right)}{\sum_{j=1}^{m} \exp \left(\vartheta_{j} / k\right)}}+\left(F_{m+1}\right)^{\frac{\exp \left(\vartheta_{m+1} / k\right)}{\sum_{j=1}^{m+1} \exp \left(\vartheta_{j} / k\right)}} \\
& \left.-\prod_{j=1}^{m}\left(F_{j}\right)^{\frac{\exp \left(\vartheta_{j} / k\right)}{\sum_{j=1}^{m} \exp \left(\vartheta_{j} / k\right)}} \times\left(F_{m+1}\right)^{\frac{\exp \left(\vartheta_{m+1} / k\right)}{\sum_{j=1}^{m+1} \exp \left(\vartheta_{j} / k\right)}}\right) \\
& =\left(\prod_{j=1}^{m+1}\left(T_{j}\right)^{\frac{\exp \left(\vartheta_{j} / k\right)}{\sum_{j=1}^{m+1} \exp \left(\vartheta_{j} / k\right)}}, \quad 1-\prod_{j=1}^{m+1}\left(1-I_{j}\right)^{\frac{\exp \left(\vartheta_{j} / k\right)}{\sum_{j=1}^{m+1} \exp \left(\vartheta_{j} / k\right)}}\right. \\
& \left.1-\prod_{j=1}^{m+1}\left(1-F_{j}\right)^{\frac{\exp \left(\vartheta_{j} / k\right)}{\sum_{j=1}^{m+1} \exp \left(\vartheta_{j} / k\right)}}\right)
\end{aligned}
$$

Therefore, Eq. (7) holds for $n=m+1$, hence the Eq. (7) holds for all positive integer by principle of mathematical induction. Hence, the proof of the theorem is completed.

Example 2 Let $\alpha_{1}=(0.6,0.4,0.5), \alpha_{2}=(0.7,0.3,0.5), \alpha_{3}=(0.8,0.3,0.4)$ and $\alpha_{4}=(0.7,0.4,0.5)$ be the four SVN-numbers. Rank the four SVN-numbers using $S S V N W G$ operator.

Solution: In the following, we use the $S S V N W G$ operator to aggregate these SVN-numbers. At first we have calculated the score values of four SVNNs using Eq. (1)

$S\left(\alpha_{1}\right)=0.567, S\left(\alpha_{2}\right)=0.633, S\left(\alpha_{3}\right)=0.700, S\left(\alpha_{4}\right)=0.600$

then $\vartheta_{1}=1, \vartheta_{2}=0.567, \vartheta_{3}=0.359, \vartheta_{4}=0.251$.

To calculate the $\exp \left(\vartheta_{j} / k\right)$ we take $k=1$, then

$\exp \left(\vartheta_{1} / k\right)=2.718, \exp \left(\vartheta_{2} / k\right)=1.763, \exp \left(\vartheta_{3} / k\right)=1.432$,

$\exp \left(\vartheta_{4} / k\right)=1.285$ and

$$
\begin{aligned}
& \frac{\exp \left(\vartheta_{1} / k\right)}{\sum_{j=1}^{4} \exp \left(\vartheta_{j} / k\right)}=0.378, \frac{\exp \left(\vartheta_{2} / k\right)}{\sum_{j=1}^{4} \exp \left(\vartheta_{j} / k\right)}=0.245, \\
& \frac{\exp \left(\vartheta_{3} / k\right)}{\sum_{j=1}^{4} \exp \left(\vartheta_{j} / k\right)}=0.199, \frac{\exp \left(\vartheta_{4} / k\right)}{\sum_{j=1}^{4} \exp \left(\vartheta_{j} / k\right)}=0.178,
\end{aligned}
$$

$$
\begin{aligned}
S S V N W G\left(\alpha_{1}, \alpha_{2}, \alpha_{3}, \alpha_{4}\right)= & \left((0.6)^{0.378} \times(0.7)^{0.245} \times(0.8)^{0.199} \times(0.7)^{0.178},\right. \\
& 1-(1-0.4)^{0.378} \times(1-0.3)^{0.245} \times(1-0.3)^{0.199} \times(1-0.4)^{0.178}, \\
& \left.1-(1-0.5)^{0.378} \times(1-0.5)^{0.245} \times(1-0.4)^{0.199} \times(1-0.5)^{0.178}\right) \\
= & (0.678,0.357,0.482) .
\end{aligned}
$$




\subsubsection{Properties of SSVNWG operator}

\section{Property 1: Idem-potency}

If $\alpha_{1}=\alpha_{2}=\ldots=\alpha_{n}=\alpha($ say $)$, then

theSSVNWG $\left(\alpha_{1}, \alpha_{2}, \ldots, \alpha_{n}\right)=\alpha$

Proof : Let $\alpha_{j}=\left\langle T_{j}, I_{j}, F_{j}\right\rangle,(j=1,2,3 \ldots, n)$ and $\alpha=\langle T, I, F\rangle$.

Since all $\alpha_{j}$ are equal, based on Theorem (2), we get

$\operatorname{SSVNWG(\alpha ,\alpha ,\ldots ,\alpha )}$

$=\left((T)^{\sum_{j=1}^{n} \phi_{k}^{j}}, 1-(1-I)^{\sum_{j=1}^{n} \phi_{k}^{j}}, 1-(1-F)^{\sum_{j=1}^{n} \phi_{k}^{j}}\right)$

$=\langle T, I, F\rangle=\alpha$.

Since, $\phi_{k}^{j}=\frac{e x p\left(\vartheta_{j} / k\right)}{\sum_{j=1}^{n} \exp \left(\vartheta_{j} / k\right)}, k>0$ and $\sum_{j=1}^{n} \phi_{k}^{j}=1$. Hence the proof is completed.

\section{Property 2: Monotonicity}

Let $\alpha_{j}(j=1,2, \ldots, n)$ and $\beta_{j}(j=1,2, \ldots, n)$ be any two sets of SVN-numbers. If $\alpha_{j} \leq \beta_{j}$ for any $j$, then $S S V N W G\left(\alpha_{1}, \alpha_{2}, \ldots, \alpha_{n}\right) \leq S S V N W G\left(\beta_{1}, \beta_{2}, \ldots, \beta_{n}\right)$.

Proof : Based on the Theorem (2), we get

$$
\begin{aligned}
S S V N W G\left(\alpha_{1}, \alpha_{2}, \ldots, \alpha_{n}\right)= & \left(\prod_{j=1}^{n}\left(T_{\alpha_{j}}\right)^{\frac{\exp \left(\vartheta \vartheta_{j} / k\right)}{\sum_{j=1}^{n} \exp \left(\vartheta_{j} / k\right)}},\right. \\
& \left.\left(1-\prod_{j=1}^{n}\left(1-I_{\alpha_{j}}\right)^{\frac{e x p\left(\vartheta \alpha_{j} / k\right)}{\sum_{j=1}^{n} \exp \left(\vartheta_{\alpha_{j}} / k\right)}}\right),\left(1-\prod_{j=1}^{n}\left(1-T_{\alpha_{j}}\right)^{\frac{e x p\left(\vartheta \alpha_{j} / k\right)}{\sum_{j=1}^{n} \exp \left(\vartheta \alpha_{j} / k\right)}}\right)\right)
\end{aligned}
$$

and

$$
\begin{aligned}
S S V N W A\left(\beta_{1}, \beta_{2}, \ldots, \beta_{n}\right)= & \left(\prod_{j=1}^{n}\left(T_{\beta_{j}}\right)^{\left.\frac{\exp \left(\vartheta_{\beta_{j}} / k\right)}{\sum_{j=1}^{n} \operatorname{exp(\vartheta _{\beta _{j}}/k)}}\right),}\right. \\
& \left(1-\prod_{j=1}^{n}\left(1-I_{\beta_{j}}\right)^{\frac{\exp \left(\vartheta_{\beta_{j}} / k\right)}{\sum_{j=1}^{n} \operatorname{exp(\vartheta _{\beta _{j}}/k)}}},\left(1-\prod_{j=1}^{n}\left(1-F_{\beta_{j}}\right)^{\frac{\left.\exp _{j=1}^{n} \vartheta_{\beta_{j}} / k\right)}{\sum^{n}\left(\vartheta_{\beta_{j}} / k\right)}}\right)\right)
\end{aligned}
$$

Since all $\alpha_{j} \leq \beta_{j}(j=1,2, \ldots, n)$. Therefore,

$$
\begin{aligned}
& T_{\alpha_{j}} \leq T_{\beta_{j}} \Rightarrow\left(T_{\alpha_{j}}\right)^{\frac{\exp \left(\vartheta_{\alpha_{j}} / k\right)}{\sum_{j=1}^{n} \exp \left(\vartheta \alpha_{j} / k\right)}} \leq\left(T_{\beta_{j}}\right)^{\frac{\exp \left(\vartheta_{\beta_{j}} / k\right)}{\sum_{j=1}^{n} \exp \left(\vartheta_{\beta_{j}} / k\right)}} \\
& \Rightarrow \prod_{j=1}^{n}\left(T_{\alpha_{j}}\right)^{\frac{\exp \left(\vartheta_{\alpha_{j}} / k\right)}{\sum_{j=1}^{n} \exp \left(\vartheta_{\alpha_{j}} / k\right)}} \leq \prod_{j=1}^{n}\left(T_{\beta_{j}}\right)^{\frac{\exp \left(\vartheta_{\beta_{j}} / k\right)}{\sum_{j=1}^{n} \exp \left(\vartheta_{\beta_{j}} / k\right)}}
\end{aligned}
$$

Further,

$$
\begin{aligned}
& I_{\alpha_{j}} \geq I_{\beta_{j}} \Rightarrow\left(1-I_{\alpha_{j}}\right) \leq\left(1-I_{\beta_{j}}\right) \\
& \Rightarrow\left(1-I_{\alpha_{j}}\right)^{\frac{\exp \left(\vartheta_{\alpha_{j}} / k\right)}{\sum_{j=1}^{n} \operatorname{exp(\vartheta \alpha _{j}/k)}}} \leq\left(1-I_{\beta_{j}}\right)^{\frac{\exp \left(\vartheta_{\beta_{j}} / k\right)}{\sum_{j=1}^{n} \exp \left(\vartheta_{\beta_{j}} / k\right)}} \\
& \Rightarrow \prod_{j=1}^{n}\left(1-I_{\alpha_{j}}\right)^{\frac{\exp \left(\vartheta_{\alpha_{j}} / k\right)}{\left.\sum_{j=1}^{n} \operatorname{exp(\vartheta } \alpha_{j} / k\right)}} \leq \prod_{j=1}^{n}\left(1-I_{\beta_{j}}\right)^{\frac{\exp \left(\vartheta_{\beta_{j}} / k\right)}{\sum_{j=1}^{n} \exp \left(\vartheta_{\beta_{j}} / k\right)}} \\
& \Rightarrow\left(1-\prod_{j=1}^{n}\left(1-I_{\alpha_{j}}\right)^{\frac{\exp \left(\vartheta \alpha_{j} / k\right)}{\sum_{j=1}^{n} \operatorname{exp(\vartheta \alpha _{j}/k)}}}\right) \geq\left(1-\prod_{j=1}^{n}\left(1-I_{\beta_{j}}\right)^{\frac{\exp \left(\vartheta_{\beta_{j}} / k\right)}{\left.\sum_{j=1}^{n} \operatorname{exp(\vartheta \beta _{j}} / k\right)}}\right)
\end{aligned}
$$


Similarly, we have also

$$
\Rightarrow\left(1-\prod_{j=1}^{n}\left(1-F_{\alpha_{j}}\right)^{\frac{\exp \left(\vartheta_{\alpha_{j}} / k\right)}{\sum_{j=1}^{n} \operatorname{exp(\vartheta \alpha _{j}/k)}}}\right) \geq\left(1-\prod_{j=1}^{n}\left(1-F_{\beta_{j}}\right)^{\frac{\exp \left(\vartheta_{\beta_{j}} / k\right)}{\sum_{j=1}^{n} \exp \left(\vartheta_{\beta_{j}} / k\right)}}\right)
$$

Hence the proof is completed.

\section{Property 3: Boundedness}

Let $\alpha_{j}(j=1,2, \ldots, n)$ be any set of SVN-number. If $\alpha^{-}=\min \left\{\alpha_{j}\right\}$ and $\alpha^{+}=\max \left\{\alpha_{j}\right\}$, then

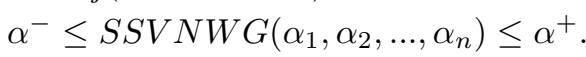

Proof : Let $\alpha^{+}=\max \left\{\alpha_{1}, \alpha_{2}, \ldots, \alpha_{n}\right\}$ and $\alpha^{-}=\min \left\{\alpha_{1}, \alpha_{2}, \ldots, \alpha_{n}\right\}$.

According to properties 1 and 2 , we have

$\operatorname{SSVNWG}\left(\alpha_{1}, \alpha_{2}, \ldots, \alpha_{n}\right) \geq \operatorname{SSVNWG}\left(\alpha^{-}, \alpha^{-}, \ldots, \alpha^{-}\right)=\alpha^{-}$and

$S S V N W G\left(\alpha_{1}, \alpha_{2}, \ldots, \alpha_{n}\right) \leq S S V N W G\left(\alpha^{+}, \alpha^{+}, \ldots, \alpha^{+}\right)=\alpha^{+}$

So we have $\alpha^{-} \leq S S V N W G\left(\alpha_{1}, \alpha_{2}, \ldots, \alpha_{n}\right) \leq \alpha^{+}$.

Hence the proof is completed.

Definition 3.6 Let $\alpha_{j}(j=1,2, \ldots, n)$ be a collection of SVN-numbers. Then generalized softmax single valued neutrosophic weighted average (GSSVNWA) operator is a function $\alpha^{n} \rightarrow \alpha$ such that

$$
G S S V N W A\left(\alpha_{1}, \alpha_{2}, \ldots, \alpha_{n}\right)=\left(\oplus_{j=1}^{n} \phi_{k}^{j} \alpha_{j}^{\lambda}\right)^{\frac{1}{\lambda}}=\left(\phi_{k}^{1} \alpha_{1}^{\lambda} \oplus \phi_{k}^{2} \alpha_{2}^{\lambda} \oplus \ldots \oplus \phi_{k}^{n} \alpha_{n}^{\lambda}\right)^{\frac{1}{\lambda}}
$$

Definition 3.7 Let $\alpha_{j}(j=1,2, \ldots, n)$ be a collection of SVN-numbers. Then generalized softmax single valued neutrosophic weighted geometric (GSSVNWG) operator is a function $\alpha^{n} \rightarrow \alpha$ such that

$$
G S S V N W G\left(\alpha_{1}, \alpha_{2}, \ldots, \alpha_{n}\right)=\frac{1}{\lambda}\left(\otimes_{j=1}^{n}\left(\lambda \phi_{j}\right)^{\phi_{k}^{j}}\right)=\frac{1}{\lambda}\left(\left(\lambda \alpha_{1}\right)^{\phi_{k}^{1}} \otimes\left(\lambda \alpha_{2}\right)^{\phi_{k}^{2}} \otimes \ldots \otimes\left(\lambda \alpha_{n}\right)^{\phi_{k}^{n}}\right)
$$

Theorem 3 Let $\alpha_{j}(j=1,2, \ldots, n)$, be a collection of SVN-numbers, then aggregated value of SVN-numbers using the GSSVNWA operation is also a SVNN, and

$$
\begin{array}{r}
G S S N W A\left(\alpha_{1}, \alpha_{2}, \ldots, \alpha_{n}\right)=\left\langle\left(1-\prod_{j=1}^{n}\left(1-T_{j}^{\lambda}\right)^{\frac{\exp \left(\vartheta_{j} / k\right)}{\sum_{j=1}^{n} \exp \left(\vartheta_{j} / k\right)}}\right)^{\frac{1}{\lambda}}, 1-\left(1-\prod_{j=1}^{n}\left(1-\left(1-I_{j}\right)^{\lambda}\right)^{\frac{\exp \left(\vartheta_{j} / k\right)}{\sum_{j=1}^{n} \exp \left(\vartheta_{j} / k\right)}}\right)^{\frac{1}{\lambda}},\right. \\
1-\left(1-\prod_{j=1}^{n}\left(1-\left(1-F_{j}\right)^{\lambda}\right)^{\left.\frac{\exp \left(\vartheta_{j} / k\right)}{\left.\sum_{j=1}^{n} \exp _{j} / k\right)}\right)^{\frac{1}{\lambda}}}\right\rangle(10)
\end{array}
$$

Proof : We proof the above theorem 3 by using mathematical induction.

For $n=1$, we have:

$$
\begin{aligned}
& G S S V N W A\left(\alpha_{1}, \alpha_{2}, \ldots, \alpha_{n}\right)=\left\langle\left(1-\prod_{j=1}^{1}\left(1-T_{j}^{\lambda}\right)^{\frac{\exp \left(\vartheta_{j} / k\right)}{\sum_{j=1}^{1} \exp \left(\vartheta_{j} / k\right)}}\right)^{\frac{1}{\lambda}}, 1-\left(1-\prod_{j=1}^{1}\left(1-\left(1-I_{j}\right)^{\lambda}\right)^{\left.\frac{\exp \left(\vartheta_{j} / k\right)}{\sum_{j=1}^{1} \operatorname{exp(\vartheta _{j}/k)}}\right)^{\frac{1}{\lambda}}}\right.\right. \\
&\left.1-\left(1-\prod_{j=1}^{1}\left(1-\left(1-F_{j}\right)^{\lambda}\right)^{\frac{\exp \left(\vartheta_{j} / k\right)}{\sum_{j=1}^{1} \exp \left(\vartheta_{j} / k\right)}}\right)^{\frac{1}{\lambda}}\right\rangle \\
&=\left\langle T_{1}, I_{1}, F_{1}\right\rangle
\end{aligned}
$$


Since, for $n=1, \frac{\exp \left(\vartheta_{j} / k\right)}{\sum_{j=1}^{n} \exp \left(\vartheta_{j} / k\right)}=1$.

Thus Eq.(10) holds for $n=1$, we assume that the Eq. (10) holds for $n=m$,

$$
\begin{array}{r}
G S S V N W\left(\alpha_{1}, \alpha_{2}, \ldots, \alpha_{m}\right)=\left\langle\left(1-\prod_{j=1}^{m}\left(1-T_{j}^{\lambda}\right)^{\frac{\exp \left(\vartheta_{j} / k\right)}{\sum_{j=1}^{m} \exp \left(\vartheta_{j} / k\right)}}\right)^{\frac{1}{\lambda}}, 1-\left(1-\prod_{j=1}^{m}\left(1-\left(1-I_{j}\right)^{\lambda}\right)^{\frac{\exp \left(\vartheta_{j} / k\right)}{\sum_{j=1}^{m} \exp \left(\vartheta_{j} / k\right)}}\right)^{\frac{1}{\lambda}},\right. \\
\left.1-\left(1-\prod_{j=1}^{m}\left(1-\left(1-F_{j}\right)^{\lambda}\right)^{\frac{\exp \left(\vartheta_{j} / k\right)}{\sum_{j=1}^{m} e^{\exp \left(\vartheta_{j} / k\right)}}}\right)^{\frac{1}{\lambda}}\right\rangle
\end{array}
$$

Now, we prove that the Eq. (10) holds for $n=m+1$.

$$
\begin{aligned}
& G S S V N W A\left(\alpha_{1}, \alpha_{2}, \ldots, \alpha_{m}, \alpha_{m+1}\right)=\left(\left(\phi_{k}^{1} \alpha_{1}^{\lambda} \oplus \phi_{k}^{2} \alpha_{2}^{\lambda} \oplus \ldots \oplus \phi_{k}^{m} \alpha_{m}^{\lambda}\right) \oplus \phi_{k}^{m+1} \alpha_{m+1}^{\lambda}\right)^{\frac{1}{\lambda}} \\
& =\left\langle\left(1-\prod_{j=1}^{m}\left(1-T_{j}^{\lambda}\right)^{\frac{\exp \left(\vartheta_{j} / k\right)}{\sum_{j=1}^{m} \exp \left(\vartheta_{j} / k\right)}}\right)^{\frac{1}{\lambda}}+\left(1-\left(1-T_{m+1}^{\lambda}\right)^{\frac{\exp \left(\vartheta_{m+1} / k\right)}{\sum_{j=1}^{m=1} \exp \left(\vartheta_{j} / k\right)}}\right)^{\frac{1}{\lambda}}\right. \\
& -\left(1-\prod_{j=1}^{m}\left(1-T_{j}^{\lambda}\right)^{\frac{\exp \left(\vartheta_{j} / k\right)}{\sum_{j=1}^{m} \exp \left(\vartheta_{j} / k\right)}}\right)^{\frac{1}{\lambda}} \times\left(1-\left(1-T_{m+1}^{\lambda}\right)^{\frac{\exp \left(\vartheta_{m+1} / k\right)}{\sum_{j=1}^{m=+1} \exp \left(\vartheta_{j} / k\right)}}\right)^{\frac{1}{\lambda}}, \\
& \left(1-\left(1-\prod_{j=1}^{m}\left(1-\left(1-I_{j}\right)^{\lambda}\right)^{\frac{\exp \left(\vartheta_{j} / k\right)}{\sum_{j=1}^{m} \exp \left(\vartheta_{j} / k\right)}}\right)^{\frac{1}{\lambda}}\right) \times \\
& \left(1-\left(1-\left(1-\left(1-I_{m+1}\right)^{\lambda}\right)^{\frac{\exp \left(\vartheta_{m+1} / k\right)}{\sum_{j=1}^{m+1} \exp \left(\vartheta_{j} / k\right)}}\right)^{\frac{1}{\lambda}}\right), \\
& \left(1-\left(1-\prod_{j=1}^{m}\left(1-\left(1-F_{j}\right)^{\lambda}\right)^{\frac{\exp \left(\vartheta_{j} / k\right)}{\sum_{j=1}^{m} \exp \left(\vartheta_{j} / k\right)}}\right)^{\frac{1}{\lambda}}\right) \times \\
& \left.\left(1-\left(1-\left(1-\left(1-F_{m+1}\right)^{\lambda}\right)^{\frac{\exp \left(\vartheta_{m+1} / k\right)}{\sum_{j=1}^{m+1} \exp \left(\vartheta_{j} / k\right)}}\right)^{\frac{1}{\lambda}}\right)\right\rangle \\
& =\left\langle\left(1-\prod_{j=1}^{m+1}\left(1-T_{j}^{\lambda}\right)^{\frac{\exp \left(\vartheta_{j} / k\right)}{\sum_{j=1}^{m+1} \exp \left(\vartheta_{j} / k\right)}}\right)^{\frac{1}{\lambda}},\right. \\
& 1-\left(1-\prod_{j=1}^{m+1}\left(1-\left(1-I_{j}\right)^{\lambda}\right)^{\frac{\exp \left(\vartheta_{j} / k\right)}{\sum_{j=1}^{m+1} \exp \left(\vartheta_{j} / k\right)}}\right)^{\frac{1}{\lambda}}, \\
& \left.1-\left(1-\prod_{j=1}^{m+1}\left(1-\left(1-F_{j}\right)^{\lambda}\right)^{\frac{\exp \left(\vartheta_{j} / k\right)}{\sum_{j=1}^{m+1} \exp \left(\vartheta_{j} / k\right)}}\right)^{\frac{1}{\lambda}}\right\rangle
\end{aligned}
$$

Therefore, Eq. (10) holds for $n=m+1$, hence the Eq. (10) holds for all positive integer by principle of mathematical induction. Hence the proof of the theorem is completed.

Example 3 Let $\alpha_{1}=(0.6,0.4,0.5), \alpha_{2}=(0.7,0.3,0.5), \alpha_{3}=(0.8,0.3,0.4)$ and $\alpha_{4}=(0.7,0.4,0.5)$ be the four SVN-numbers. Rank the four SVN-numbers using the GSSVNWA operator. 
Solution: In the following, we use the GSSVNWA operator to aggregate these SVN-numbers. At first we calculate the score values of four SVN-numbers using Eq. (1). $S\left(\alpha_{1}\right)=0.567, S\left(\alpha_{2}\right)=0.633, S\left(\alpha_{3}\right)=0.700, S\left(\alpha_{4}\right)=$ 0.600 then $\vartheta_{1}=1, \vartheta_{2}=0.567, \vartheta_{3}=0.359, \vartheta_{4}=0.251$. To calculate $\exp \left(\vartheta_{j} / k\right)$ we take $k=1$, then

$$
\exp \left(\vartheta_{1} / k\right)=2.718, \exp \left(\vartheta_{2} / k\right)=1.763, \exp \left(\vartheta_{3} / k\right)=1.432, \exp \left(\vartheta_{4} / k\right)=1.285
$$

and

$$
\begin{aligned}
& \frac{\exp \left(\vartheta_{1} / k\right)}{\sum_{j=1}^{4} \exp \left(\vartheta_{j} / k\right)}=0.378, \frac{\exp \left(\vartheta_{2} / k\right)}{\sum_{j=1}^{4} \exp \left(\vartheta_{j} / k\right)}=0.245, \\
& \frac{\exp \left(\vartheta_{3} / k\right)}{\sum_{j=1}^{4} \exp \left(\vartheta_{j} / k\right)}=0.199, \frac{\exp \left(\vartheta_{4} / k\right)}{\sum_{j=1}^{4} \exp \left(\vartheta_{j} / k\right)}=0.178
\end{aligned}
$$

Taking $\lambda=1$ GSSVNWA reduces to SSVNWA and we get

$$
\operatorname{GSSVNWA}\left(\alpha_{1}, \alpha_{2}, \alpha_{3}, \alpha_{4}\right)=(0.691,0.352,0.478) .
$$

Taking $\lambda=2$ we obtain

$$
\begin{aligned}
& \operatorname{GSSVNWA}\left(\alpha_{1}, \alpha_{2}, \alpha_{3}, \alpha_{4}\right)=\left(\left(1-\left(1-(0.6)^{2}\right)^{0.378} \times\left(1-(0.7)^{2}\right)^{0.245} \times\left(1-(0.8)^{2}\right)^{0.199} \times\left(1-(0.7)^{2}\right)^{0.178}\right)^{\frac{1}{2}},\right. \\
& 1-\left(1-\left(1-(1-0.6)^{2}\right)^{0.378} \times\left(1-(1-0.3)^{2}\right)^{0.245} \times\right. \\
& \left.\left(1-(1-0.3)^{2}\right)^{0.199} \times\left(1-(1-0.4)^{2}\right)^{0.178}\right)^{\frac{1}{2}} \text {, } \\
& 1-\left(1-\left(1-(1-0.5)^{2}\right)^{0.378} \times\left(1-(1-0.5)^{2}\right)^{0.245} \times\right. \\
& \left.\left.\left(1-(1-0.4)^{2}\right)^{0.199} \times\left(1-(1-0.5)^{2}\right)^{0.178}\right)^{\frac{1}{2}}\right) \\
& =(0.694,0.401,0.477)
\end{aligned}
$$

Taking $\lambda=3$, we obtain

$$
\begin{aligned}
& \operatorname{GSSVNWA}\left(\alpha_{1}, \alpha_{2}, \alpha_{3}, \alpha_{4}\right)=\left(\left(1-\left(1-(0.6)^{3}\right)^{0.378} \times\left(1-(0.7)^{3}\right)^{0.245} \times\left(1-(0.8)^{3}\right)^{0.199} \times\left(1-(0.7)^{3}\right)^{0.178}\right)^{\frac{1}{3}},\right. \\
& 1-\left(1-\left(1-(1-0.6)^{3}\right)^{0.378} \times\left(1-(1-0.3)^{3}\right)^{0.245} \times\right. \\
& \left.\left(1-(1-0.3)^{3}\right)^{0.199} \times\left(1-(1-0.4)^{3}\right)^{0.178}\right)^{\frac{1}{3}} \text {, } \\
& 1-\left(1-\left(1-(1-0.5)^{3}\right)^{0.378} \times\left(1-(1-0.5)^{3}\right)^{0.245} \times\right. \\
& \left.\left.\left(1-(1-0.4)^{3}\right)^{0.199} \times\left(1-(1-0.5)^{3}\right)^{0.178}\right)^{\frac{1}{3}}\right) \\
& =(0.697,0.392,0.476)
\end{aligned}
$$

Similarly we can also checked for $\lambda=4,5, \ldots$ and so on

3.7.1 Properties of GSSVNWA operator

Property 1: Idem-potency If $\alpha_{1}=\alpha_{2}=\ldots=\alpha_{n}=\alpha$ (say), then the GSSVNWAO( $\left.\alpha_{1}, \alpha_{2}, \ldots, \alpha_{n}\right)=\alpha$ 
Proof Let $\alpha_{j}=\left\langle T_{j}, I_{j}, F_{j}\right\rangle,(j=1,2,3 \ldots, n)$ and $\alpha=\langle T, I, F\rangle$.

Since all $\alpha_{j}$ are equal, based on Theorem (3), we get

$$
\begin{aligned}
& S S V N W G(\alpha, \alpha, \ldots, \alpha)=\left\langle\left(1-\left(1-T^{\lambda}\right)^{\sum_{j=1}^{n} \phi_{k}^{j}}\right)^{\frac{1}{\lambda}}, 1-\left(1-\left(1-(1-I)^{\lambda}\right)^{\sum_{j=1}^{n} \phi_{k}^{j}}\right)^{\frac{1}{\lambda}},\right. \\
& \left.1-\left(1-\left(1-(1-F)^{\lambda}\right)^{\sum_{j=1}^{n} \phi_{k}^{j}}\right)^{\frac{1}{\lambda}}\right\rangle \\
& =\langle T, I, F\rangle=\alpha
\end{aligned}
$$

Since, $\phi_{k}^{j}=\frac{\exp \left(\vartheta_{j} / k\right)}{\sum_{j=1}^{n} \exp \left(\vartheta_{j} / k\right)}, k>0$ and $\sum_{j=1}^{n} \phi_{k}^{j}=1$.

Hence the proof is completed.

\section{Property 2: Monotonicity}

Let $\alpha_{j}(j=1,2, \ldots, n)$ and $\beta_{j}(j=1,2, \ldots, n)$ be any two SVN-numbers. If $\alpha_{j} \leq \beta_{j}$ for any $j$, then GSSVNWA $\left(\alpha_{1}, \alpha_{2}, \ldots, \alpha_{n}\right) \leq G S S V N W A\left(\beta_{1}, \beta_{2}, \ldots, \beta_{n}\right)$.

Proof : Based on the Theorem (3), we get

$$
\begin{aligned}
& G S S V N W A\left(\alpha_{1}, \alpha_{2}, \ldots, \alpha_{n}\right)=\left\langle\left(1-\prod_{j=1}^{n}\left(1-T_{\alpha_{j}}^{\lambda}\right)^{\frac{\exp \left(\vartheta_{\alpha_{j}} / k\right)}{\sum_{j=1}^{n} \exp \left(\vartheta_{\alpha_{j}} / k\right)}}\right)^{\frac{1}{\lambda}}\right. \\
& 1-\left(1-\prod_{j=1}^{n}\left(1-\left(1-I_{\alpha_{j}}\right)^{\lambda}\right)^{\frac{\exp \left(\vartheta_{\alpha_{j}} / k\right)}{\sum_{j=1}^{n} \exp \left(\vartheta_{\alpha_{j}} / k\right)}}\right)^{\frac{1}{\lambda}}, \\
& 1-\left(1-\prod_{j=1}^{n}\left(1-\left(1-F_{\alpha_{j}}\right)^{\lambda}\right)^{\frac{e x p\left(\vartheta_{\alpha_{j}} / k\right)}{\sum_{j=1}^{n} \exp \left(\vartheta_{\alpha_{j}} / k\right)}}\right)^{\frac{1}{\lambda}}
\end{aligned}
$$

and

$$
\begin{aligned}
& G S S V N W A\left(\beta_{1}, \beta_{2}, \ldots, \beta_{n}\right)=\left\langle\left(1-\prod_{j=1}^{n}\left(1-T_{\beta_{j}}^{\lambda}\right)^{\frac{\exp \left(\vartheta_{\beta_{j}} / k\right)}{\sum_{j=1}^{n} \exp _{\left(\vartheta_{\beta_{j}} / k\right)}}}\right)^{\frac{1}{\lambda}},\right. \\
& 1-\left(1-\prod_{j=1}^{n}\left(1-\left(1-I_{\beta_{j}}\right)^{\lambda}\right)^{\frac{\exp \left(\vartheta_{\beta_{j}} / k\right)}{\sum_{j=1}^{n} \exp \left(\vartheta_{\beta_{j}} / k\right)}}\right)^{\frac{1}{\lambda}}, \\
& \left.1-\left(1-\prod_{j=1}^{n}\left(1-\left(1-F_{\beta_{j}}\right)^{\lambda}\right)^{\sum_{j=1}^{n}{\exp \left(\vartheta_{\beta_{j}} / k\right)}^{\exp \left(\vartheta_{\beta_{j}} / k\right)}}\right)^{\frac{1}{\lambda}}\right\rangle
\end{aligned}
$$


Since all $\alpha_{j} \leq \beta_{j}(j=1,2, \ldots, n)$.

Therefore for $T_{\alpha_{j}} \leq T_{\beta_{j}}$ we have

$$
\begin{aligned}
& T_{\alpha_{j}}^{\lambda} \leq T_{\beta_{j}}^{\lambda} \Rightarrow\left(1-T_{\alpha_{j}}^{\lambda}\right) \geq\left(1-T_{\beta_{j}}^{\lambda}\right) \\
& \Rightarrow\left(1-T_{\alpha_{j}}^{\lambda}\right)^{\frac{\exp _{j=1}\left(\vartheta_{\alpha_{j}} / k\right)}{\sum_{j=1}^{n}\left(\vartheta_{\left.\alpha_{j} / k\right)}\right.}} \geq\left(1-T_{\beta_{j}}^{\lambda}\right)^{\frac{\exp \left(\vartheta_{\beta_{j}} / k\right)}{\sum_{j=1}^{n} \exp \left(\vartheta_{\beta_{j}} / k\right)}} \\
& \Rightarrow \prod_{j=1}^{n}\left(1-T_{\alpha_{j}}^{\lambda}\right)^{\frac{\exp \left(\vartheta_{\alpha_{j}} / k\right)}{\sum_{j=1}^{n} \exp \left(\vartheta_{\alpha_{j}} / k\right)}} \geq \prod_{j=1}^{n}\left(1-T_{\beta_{j}}^{\lambda}\right)^{\frac{\exp \left(\vartheta_{\beta_{j}} / k\right)}{\sum_{j=1}^{n} \exp \left(\vartheta_{\beta_{j}} / k\right)}} \\
& \Rightarrow\left(1-\prod_{j=1}^{n}\left(1-T_{\alpha_{j}}^{\lambda}\right)^{\frac{\exp \left(\vartheta_{\alpha_{j}} / k\right)}{\sum_{j=1}^{n} \exp \left(\vartheta_{\alpha_{j}} / k\right)}}\right) \leq\left(1-\prod_{j=1}^{n}\left(1-T_{\beta_{j}}^{\lambda}\right)^{\frac{\exp \left(\vartheta_{\beta_{j}} / k\right)}{\sum_{j=1}^{n} \exp \left(\vartheta_{\beta_{j}} / k\right)}}\right) \\
& \Rightarrow\left(1-\prod_{j=1}^{n}\left(1-T_{\alpha_{j}}^{\lambda}\right)^{\frac{\exp \left(\vartheta_{\alpha_{j}} / k\right)}{\sum_{j=1}^{n} \exp \left(\vartheta_{\alpha_{j}} / k\right)}}\right)^{\frac{1}{\lambda}} \leq\left(1-\prod_{j=1}^{n}\left(1-T_{\beta_{j}}^{\lambda}\right)^{\frac{\exp \left(\vartheta_{\beta_{j}} / k\right)}{\sum_{j=1}^{n} \exp _{\left(\vartheta_{\beta_{j}} / k\right)}}}\right)^{\frac{1}{\lambda}}
\end{aligned}
$$

Further for

$$
\begin{aligned}
& I_{\alpha_{j}} \geq I_{\beta_{j}} \Rightarrow\left(1-I_{\alpha_{j}}\right) \leq\left(1-I_{\beta_{j}}\right) \\
& \Rightarrow\left(1-I_{\alpha_{j}}\right)^{\lambda} \leq\left(1-I_{\beta_{j}}\right)^{\lambda} \\
& \Rightarrow\left(1-\left(1-I_{\alpha_{j}}\right)^{\lambda}\right) \geq\left(1-\left(1-I_{\beta_{j}}\right)^{\lambda}\right) \\
& \Rightarrow\left(1-\left(1-I_{\alpha_{j}}\right)^{\lambda}\right)^{\frac{\exp \left(\vartheta_{\alpha_{j}} / k\right)}{\sum_{j=1}^{n} \exp \left(\vartheta_{\alpha_{j}} / k\right)}} \geq\left(1-\left(1-I_{\beta_{j}}\right)^{\lambda}\right)^{\frac{\exp \left(\vartheta_{\beta_{j}} / k\right)}{\sum_{j=1}^{n} \exp \left(\vartheta_{\beta_{j}} / k\right)}} \\
& \Rightarrow \prod_{j=1}^{n}\left(1-\left(1-I_{\alpha_{j}}\right)^{\lambda}\right)^{\frac{\exp \left(\vartheta_{\alpha_{j}} / k\right)}{\sum_{j=1}^{n} \exp \left(\vartheta_{\alpha_{j}} / k\right)}} \geq \prod_{j=1}^{n}\left(1-\left(1-I_{\beta_{j}}\right)^{\lambda}\right)^{\frac{\exp \left(\vartheta_{\beta_{j}} / k\right)}{\sum_{j=1}^{n} \exp \left(\vartheta_{\beta_{j}} / k\right)}} \\
& \Rightarrow\left(1-\prod_{j=1}^{n}\left(1-\left(1-I_{\alpha_{j}}\right)^{\lambda}\right)^{\frac{\exp \left(\vartheta_{\alpha_{j}} / k\right)}{\sum_{j=1}^{n} \exp \left(\vartheta_{\alpha_{j}} / k\right)}}\right)^{\frac{1}{\lambda}} \leq\left(1-\prod_{j=1}^{n}\left(1-\left(1-I_{\beta_{j}}\right)^{\lambda}\right)^{\frac{\exp _{j=1}^{n}\left(\vartheta_{\beta_{j}} / k\right)}{\sum_{j=1}^{\exp \left(\vartheta_{\beta_{j}} / k\right)}}}\right)^{\frac{1}{\lambda}} \\
& \Rightarrow 1-\left(1-\prod_{j=1}^{n}\left(1-\left(1-I_{\alpha_{j}}\right)^{\lambda}\right)^{\sum_{j=1}^{n} e^{e x p\left(\vartheta_{\left.\alpha_{j} / k\right)} / \vartheta_{\alpha_{j}} / k\right)}}\right)^{\frac{1}{\lambda}} \geq 1-\left(1-\prod_{j=1}^{n}\left(1-\left(1-I_{\beta_{j}}\right)^{\lambda}\right)^{\sum_{j=1}^{n} \exp ^{\exp \left(\vartheta_{\beta_{j}} / k\right)}}\right)^{\frac{1}{\lambda}}
\end{aligned}
$$

Similarly, we can also show that

$$
\Rightarrow 1-\left(1-\prod_{j=1}^{n}\left(1-\left(1-F_{\alpha_{j}}\right)^{\lambda}\right)^{\sum_{j=1}^{n} e^{\exp \left(\vartheta_{\alpha_{j}} / k\right)}\left(\vartheta_{\left.\alpha_{j} / k\right)}\right.}\right)^{\frac{1}{\lambda}} \geq 1-\left(1-\prod_{j=1}^{n}\left(1-\left(1-F_{\beta_{j}}\right)^{\lambda}\right)^{\sum_{j=1}^{n} \exp ^{\exp \left(\vartheta_{\beta_{j}} / k\right)}}\right)^{\frac{1}{\lambda}}
$$

Hence the proof is completed.

\section{Property 3: Boundedness}

Let $\alpha_{j}(j=1,2, \ldots, n)$ be any set of SVNN. If $\alpha^{-}=\min \left\{\alpha_{j}\right\}$ and $\alpha^{+}=\max \left\{\alpha_{j}\right\}$,

then $\alpha^{-} \leq G S S V N W A\left(\alpha_{1}, \alpha_{2}, \ldots, \alpha_{n}\right) \leq \alpha^{+}$.

Proof : Let $\alpha^{+}=\max \left\{\alpha_{1}, \alpha_{2}, \ldots, \alpha_{n}\right\}$ and $\alpha^{-}=\min \left\{\alpha_{1}, \alpha_{2}, \ldots, \alpha_{n}\right\}$. According to properties 1 and 2 , we have $\operatorname{GSSVNWA}\left(\alpha_{1}, \alpha_{2}, \ldots, \alpha_{n}\right) \geq \operatorname{GSSVNWA}\left(\alpha^{-}, \alpha^{-}, \ldots, \alpha^{-}\right)=\alpha^{-}$and $G S S V N W A\left(\alpha_{1}, \alpha_{2}, \ldots, \alpha_{n}\right) \leq G S S V N W A\left(\alpha^{+}, \alpha^{+}, \ldots, \alpha^{+}\right)=\alpha^{+}$

So, we have $\alpha^{-} \leq G S S V N W A\left(\alpha_{1}, \alpha_{2}, \ldots, \alpha_{n}\right) \leq \alpha^{+}$.

Hence the proof is completed. 
Theorem 4 Let $\alpha_{j}(j=1,2, \ldots, n)$, be a collection of SVN-numbers. The aggregated value of SVN-numbers using the GSSVNWG operator is also a SVN-number and

$$
\begin{aligned}
& \operatorname{GSSVNWG}\left(\alpha_{1}, \alpha_{2}, \ldots, \alpha_{n}\right)=\left\langle 1-\left(1-\prod_{j=1}^{n}\left(1-\left(1-T_{j}\right)^{\lambda}\right)^{\frac{\exp \left(\vartheta_{j} / k\right)}{\sum_{j=1}^{\exp \left(\vartheta_{j} / k\right)}}}\right)^{\frac{1}{\lambda}},\left(1-\prod_{j=1}^{n}\left(1-I_{j}^{\lambda}\right)^{\frac{\exp \left(\vartheta_{j} / k\right)}{\sum_{j=1}^{n} \exp ^{\left(\vartheta_{j} / k\right)}}}\right)^{\frac{1}{\lambda}},\right. \\
& \left.\left(1-\prod_{j=1}^{n}\left(1-F_{j}^{\lambda}\right)^{\frac{e x p\left(\vartheta_{j} / k\right)}{\sum_{j=1}^{n} e^{e x p\left(\vartheta_{j} / k\right)}}}\right)^{\frac{1}{\lambda}}\right\rangle
\end{aligned}
$$

Proof : We proof the above Theorem 4 by using mathematical induction.

For $n=1$, we have:

$$
\begin{aligned}
& \operatorname{GSSVNWG}\left(\alpha_{1}, \alpha_{2}, \ldots, \alpha_{n}\right)=\left\langle 1-\left(1-\prod_{j=1}^{1}\left(1-\left(1-T_{j}\right)^{\lambda}\right)^{\frac{\exp \left(\vartheta_{j} / k\right)}{\sum_{j=1}^{1} \exp \left(\vartheta_{j} / k\right)}}\right)^{\frac{1}{\lambda}},\left(1-\prod_{j=1}^{1}\left(1-I_{j}^{\lambda}\right)^{\frac{\exp \left(\vartheta_{j} / k\right)}{\sum_{j=1}^{1} 1^{\exp \left(\vartheta_{j} / k\right)}}}\right)^{\frac{1}{\lambda}},\right. \\
& \left.\left(1-\prod_{j=1}^{1}\left(1-F_{j}^{\lambda}\right)^{\frac{\exp \left(\vartheta_{j} / k\right)}{\left.\sum_{j=1}^{1}=\exp _{j} / k\right)}}\right)^{\frac{1}{\lambda}}\right\rangle=\left\langle T_{1}, I_{1}, F_{1}\right\rangle
\end{aligned}
$$

Since, for $n=1, \frac{\exp \left(\vartheta_{j} / k\right)}{\sum_{j=1}^{n} \exp \left(\vartheta_{j} / k\right)}=1$.

Thus Eq.(11) holds for $n=1$. Assume that the Eq. (11) holds for $n=m$,

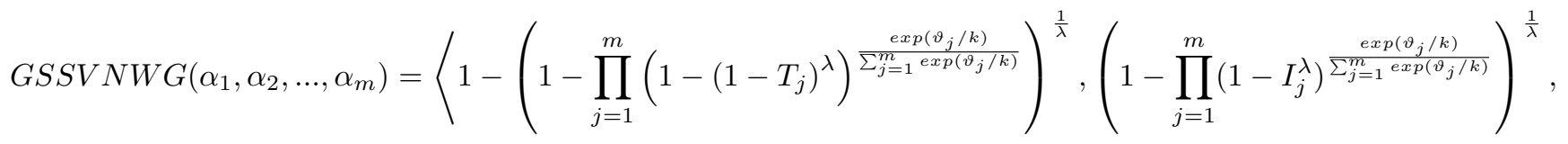

$$
\begin{aligned}
& \left.\left(1-\prod_{j=1}^{m}\left(1-F_{j}^{\lambda}\right)^{\frac{e x p\left(\vartheta_{j} / k\right)}{\sum_{j=1}^{m} \exp \left(\vartheta_{j} / k\right)}}\right)^{\frac{1}{\lambda}}\right\rangle \text {. }
\end{aligned}
$$


Now, we prove that the Eq. (12) holds for $n=m+1$.

$$
\begin{aligned}
& G S S V N W G\left(\alpha_{1}, \alpha_{2}, \ldots, \alpha_{m}, \alpha_{m+1}\right)=\frac{1}{\lambda}\left(\left(\lambda \alpha_{1}\right)^{\phi_{k}^{1}} \otimes\left(\lambda \alpha_{2}\right)^{\phi_{k}^{2}} \otimes \ldots \otimes\left(\lambda \alpha_{m}\right)^{\phi_{k}^{m}} \otimes\left(\lambda \alpha_{m+1}\right)^{\phi_{k}^{m+1}}\right) \\
& =\left\langle\left(1-\left(1-\prod_{j=1}^{m}\left(1-\left(1-T_{j}\right)^{\lambda}\right)^{\frac{\exp \left(\vartheta_{j} / k\right)}{\sum_{j=1}^{m} \exp \left(\vartheta_{j} / k\right)}}\right)^{\frac{1}{\lambda}}\right)\right. \\
& \times\left(1-\left(1-\left(1-\left(1-T_{m+1}\right)^{\lambda}\right)^{\frac{\exp \left(\vartheta_{m+1} / k\right)}{\sum_{j=1}^{m+1} \exp \left(\vartheta_{j} / k\right)}}\right)^{\frac{1}{\lambda}}\right), \\
& \left(1-\prod_{j=1}^{m}\left(1-I_{j}^{\lambda}\right)^{\frac{\exp \left(\vartheta_{j} / k\right)}{\sum_{j=1}^{m} \exp \left(\vartheta_{j} / k\right)}}\right)^{\frac{1}{\lambda}}+\left(1-\left(1-I_{m+1}^{\lambda}\right)^{\frac{\exp \left(\vartheta_{m+1} / k\right)}{\sum_{j=1}^{m+1} \exp \left(\vartheta_{j} / k\right)}}\right)^{\frac{1}{\lambda}} \\
& -\left(1-\prod_{j=1}^{m}\left(1-I_{j}^{\lambda}\right)^{\frac{\exp \left(\vartheta_{j} / k\right)}{\sum_{j=1}^{m} \exp \left(\vartheta_{j} / k\right)}}\right)^{\frac{1}{\lambda}} \times\left(1-\left(1-I_{m+1}^{\lambda}\right)^{\frac{\exp \left(\vartheta_{m+1} / k\right)}{\sum_{j=1}^{m+1} \exp \left(\vartheta_{j} / k\right)}}\right)^{\frac{1}{\lambda}}, \\
& \left(1-\prod_{j=1}^{m}\left(1-F_{j}^{\lambda}\right)^{\frac{e x p\left(\vartheta_{j} / k\right)}{\sum_{j=1}^{m=1} \exp \left(\vartheta_{j} / k\right)}}\right)^{\frac{1}{\lambda}}+\left(1-\left(1-F_{m+1}^{\lambda}\right)^{\frac{e x p(\vartheta)+1 / k)}{\sum_{j=1}^{m+1} \exp \left(\vartheta_{j} / k\right)}}\right)^{\frac{1}{\lambda}} \\
& \left.-\left(1-\prod_{j=1}^{m}\left(1-F_{j}^{\lambda}\right)^{\frac{\exp \left(\vartheta_{j} / k\right)}{\sum_{j=1}^{m} \exp \left(\vartheta_{j} / k\right)}}\right)^{\frac{1}{\lambda}} \times\left(1-\left(1-F_{m+1}^{\lambda}\right)^{\frac{\exp \left(\vartheta_{m+1} / k\right)}{\sum_{j=1}^{m+1} \exp \left(\vartheta_{j} / k\right)}}\right)^{\frac{1}{\lambda}}\right\rangle \\
& =\left\langle 1-\left(1-\prod_{j=1}^{m+1}\left(1-\left(1-T_{j}\right)^{\lambda}\right)^{\frac{\exp \left(\vartheta_{j} / k\right)}{\sum_{j=1}^{m+1} \exp \left(\vartheta_{j} / k\right)}}\right)^{\frac{1}{\lambda}},\right. \\
& \left(1-\prod_{j=1}^{m+1}\left(1-I_{j}^{\lambda}\right)^{\frac{\exp \left(\vartheta_{j} / k\right)}{\sum_{j=1}^{m+1} \exp \left(\vartheta_{j} / k\right)}}\right)^{\frac{1}{\lambda}} \\
& \left.\left(1-\prod_{j=1}^{m+1}\left(1-F_{j}^{\lambda}\right)^{\frac{\exp \left(\vartheta_{j} / k\right)}{\sum_{j=1}^{m+1} \exp \left(\vartheta_{j} / k\right)}}\right)^{\frac{1}{\lambda}}\right\rangle
\end{aligned}
$$

Therefore, Eq. (11) holds for $n=m+1$, hence the Eq. (11) holds for all positive integer by principle of mathematical induction. Hence the proof of the theorem is completed.

Example 4 Let $\alpha_{1}=(0.6,0.4,0.5), \alpha_{2}=(0.7,0.3,0.5), \alpha_{3}=(0.8,0.3,0.4)$ and $\alpha_{4}=(0.7,0.4,0.5)$ be the four SVN-numbers. Rank the four SVN-numbers using GSSVNWG operator.

Solution : In the following, we use the GSSVNWG operator to aggregate these SVN-numbers.

At first, we calculate the score values of four SVN-numbers using Eq. (1).

$S\left(\alpha_{1}\right)=0.567, S\left(\alpha_{2}\right)=0.633, S\left(\alpha_{3}\right)=0.700, S\left(\alpha_{4}\right)=0.600$, then

$\vartheta_{1}=1, \vartheta_{2}=0.567, \vartheta_{3}=0.359, \vartheta_{4}=0.251$.

To calculate $\exp \left(\vartheta_{j} / k\right)$ we take $\mathrm{k}=1$, then

$\exp \left(\vartheta_{1} / k\right)=2.718, \exp \left(\vartheta_{2} / k\right)=1.763, \exp \left(\vartheta_{3} / k\right)=1.432, \exp \left(\vartheta_{4} / k\right)=1.285$

and

$$
\begin{aligned}
& \frac{\exp \left(\vartheta_{1} / k\right)}{\sum_{j=1}^{4} \exp \left(\vartheta_{j} / k\right)}=0.378, \frac{\exp \left(\vartheta_{2} / k\right)}{\sum_{j=1}^{4} \exp \left(\vartheta_{j} / k\right)}=0.245 \\
& \frac{\exp \left(\vartheta_{3} / k\right)}{\sum_{j=1}^{4} \exp \left(\vartheta_{j} / k\right)}=0.199, \frac{\exp \left(\vartheta_{4} / k\right)}{\sum_{j=1}^{4} \exp \left(\vartheta_{j} / k\right)}=0.178,
\end{aligned}
$$




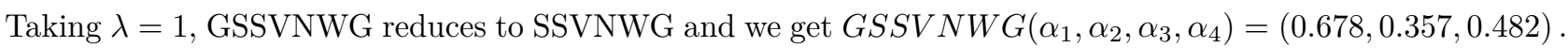
Again for $\lambda=2$, we obtain

$$
\begin{aligned}
G S S V N W G\left(\alpha_{1}, \alpha_{2}, \alpha_{3}, \alpha_{4}\right)= & \left(1-\left(1-\left(1-(1-0.6)^{2}\right)^{0.378} \times\left(1-(1-0.7)^{2}\right)^{0.245}\right.\right. \\
& \left.\times\left(1-(1-0.8)^{2}\right)^{0.199} \times\left(1-(1-0.7)^{2}\right)^{0.178}\right)^{\frac{1}{2}}, \\
& \left(1-\left(1-(0.6)^{2}\right)^{0.378} \times\left(1-(0.3)^{2}\right)^{0.245} \times\left(1-(0.3)^{2}\right)^{0.199} \times\left(1-(0.4)^{2}\right)^{0.178}\right)^{\frac{1}{2}}, \\
& \left.\left(1-\left(1-(0.5)^{2}\right)^{0.378} \times\left(1-(0.5)^{2}\right)^{0.245} \times\left(1-(0.4)^{2}\right)^{0.199} \times\left(1-(0.5)^{2}\right)^{0.178}\right)^{\frac{1}{2}}\right) \\
= & (0.671,0.464,0.482) .
\end{aligned}
$$

Taking $\lambda=3$, we obtain

$$
\begin{aligned}
G S S V N W G\left(\alpha_{1}, \alpha_{2}, \alpha_{3}, \alpha_{4}\right)= & \left(1-\left(1-\left(1-(1-0.6)^{3}\right)^{0.378} \times\left(1-(1-0.7)^{3}\right)^{0.245}\right.\right. \\
& \left.\times\left(1-(1-0.8)^{3}\right)^{0.199} \times\left(1-(1-0.7)^{3}\right)^{0.178}\right)^{\frac{1}{3}}, \\
& \left(1-\left(1-(0.6)^{3}\right)^{0.378} \times\left(1-(0.3)^{3}\right)^{0.245} \times\left(1-(0.3)^{3}\right)^{0.199} \times\left(1-(0.4)^{3}\right)^{0.178}\right)^{\frac{1}{3}}, \\
& \left.\left(1-\left(1-(0.5)^{3}\right)^{0.378} \times\left(1-(0.5)^{3}\right)^{0.245} \times\left(1-(0.4)^{3}\right)^{0.199} \times\left(1-(0.5)^{3}\right)^{0.178}\right)^{\frac{1}{3}}\right) \\
= & (0.662,0.480,0.484) .
\end{aligned}
$$

Similarly, we can also show that for $\lambda=4,5, \ldots$ and so on.

\subsubsection{Properties of GSSVNWG operator}

\section{Property 1: Idem-potency}

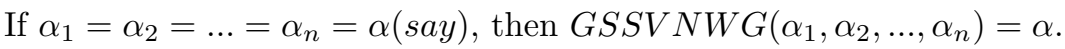

Proof Let $\alpha_{j}=\left\langle T_{j}, I_{j}, F_{j}\right\rangle,(j=1,2,3 \ldots, n)$ and $\alpha=\langle T, I, F\rangle$.

Since all $\alpha_{j}$ are equal, based on Theorem (4), we get

$$
\begin{aligned}
G S S V N W G(\alpha, \alpha, \ldots, \alpha) & =\left\langle 1-\left(1-\left(1-(1-T)^{\lambda}\right)^{\sum_{j=1}^{n} \phi_{k}^{j}}\right)^{\frac{1}{\lambda}},\left(1-\left(1-I^{\lambda}\right)^{\sum_{j=1}^{n} \phi_{k}^{j}}\right)^{\frac{1}{\lambda}},\left(1-\left(1-F^{\lambda}\right)^{\sum_{j=1}^{n} \phi_{k}^{j}}\right)^{\frac{1}{\lambda}}\right\rangle \\
& =\langle T, I, F\rangle=\alpha
\end{aligned}
$$

Since, $\phi_{k}^{j}=\frac{\exp \left(\vartheta_{j} / k\right)}{\sum_{j=1}^{n} \exp \left(\vartheta_{j} / k\right)}, k>0$ and $\sum_{j=1}^{n} \phi_{k}^{j}=1$. Hence the proof is completed.

2. Monotonicity:

Let $\alpha_{j}(j=1,2, \ldots, n)$ and $\beta_{j}(j=1,2, \ldots, n)$ be any two sets of SVN-numbers. If $\alpha_{j} \leq \beta_{j}$ for any $j$, then $G S S V N W G\left(\alpha_{1}, \alpha_{2}, \ldots, \alpha_{n}\right) \leq G S S V N W G\left(\beta_{1}, \beta_{2}, \ldots, \beta_{n}\right)$. 
Proof: Based on the Theorem (4), we get

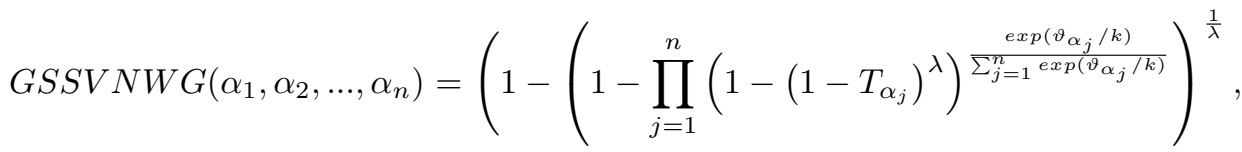

$$
\begin{aligned}
& \left(1-\prod_{j=1}^{n}\left(1-I_{\alpha_{j}}^{\lambda}\right)^{\frac{e x p\left(\vartheta_{\alpha_{j}} / k\right)}{\sum_{j=1}^{n} \exp \left(\vartheta_{\alpha_{j}} / k\right)}}\right)^{\frac{1}{\lambda}} \\
& \left.\left(1-\prod_{j=1}^{n}\left(1-F_{\alpha_{j}}^{\lambda}\right)^{\frac{\exp \left(\vartheta_{\alpha_{j}} / k\right)}{\sum_{j=1}^{n} \exp \left(\vartheta_{\alpha_{j}} / k\right)}}\right)^{\frac{1}{\lambda}}\right)
\end{aligned}
$$

and

$$
\begin{aligned}
& \operatorname{GSSVNWG}\left(\beta_{1}, \beta_{2}, \ldots, \beta_{n}\right)=\left(1-\left(1-\prod_{j=1}^{n}\left(1-\left(1-T_{\beta_{j}}\right)^{\lambda}\right)^{\frac{\exp ^{\left(\vartheta_{\beta_{j}} / k\right)}}{\sum_{j=1}^{n}{\exp \left(\vartheta_{\beta_{j}} / k\right)}}}\right)^{\frac{1}{\lambda}},\right. \\
& \left(1-\prod_{j=1}^{n}\left(1-I_{\beta_{j}}^{\lambda}\right)^{\sum_{j=1}^{n}{\exp \left(\vartheta_{\beta_{j}} / k\right)}_{\exp \left(\vartheta_{\beta_{j}} / k\right)}}\right)^{\frac{1}{\lambda}} \\
& \left.\left(1-\prod_{j=1}^{n}\left(1-F_{\beta_{j}}^{\lambda}\right)^{\frac{\exp \left(\vartheta_{\beta_{j}} / k\right)}{\left.\sum_{j=1}^{n} \exp _{\left(\vartheta_{j}\right.} / k\right)}}\right)^{\frac{1}{\lambda}}\right)
\end{aligned}
$$

Since all $\alpha_{j} \leq \beta_{j}(j=1,2, \ldots, n)$. Therefore,

$$
\begin{aligned}
& T_{\alpha_{j}} \leq T_{\beta_{j}} \Rightarrow\left(1-T_{\alpha_{j}}\right) \geq\left(1-T_{\beta_{j}}\right), \\
& \Rightarrow\left(1-T_{\alpha_{j}}\right)^{\lambda} \geq\left(1-T_{\beta_{j}}\right)^{\lambda}, \\
& \Rightarrow\left(1-\left(1-T_{\alpha_{j}}\right)^{\lambda}\right) \leq\left(1-\left(1-T_{\beta_{j}}\right)^{\lambda}\right) \\
& \Rightarrow\left(1-\left(1-T_{\alpha_{j}}\right)^{\lambda}\right)^{\frac{\exp \left(\vartheta_{\alpha_{j}} / k\right)}{\sum_{j=1}^{n} \exp \left(\vartheta_{\alpha_{j}} / k\right)}} \leq\left(1-\left(1-T_{\beta_{j}}\right)^{\lambda}\right)^{\frac{\exp \left(\vartheta_{\beta_{j}} / k\right)}{\sum_{j=1}^{n} \exp \left(\vartheta_{\beta_{j}} / k\right)}} \\
& \Rightarrow \prod_{j=1}^{n}\left(1-\left(1-T_{\alpha_{j}}\right)^{\lambda}\right)^{\sum_{j=1}^{n} e^{n x p\left(\vartheta_{\alpha_{j}} / k\right)}} \leq \prod_{j=1}^{n}\left(1-\left(1-T_{\beta_{j}}\right)^{\lambda}\right)^{\frac{\exp _{j}\left(\vartheta_{\beta_{j}} / k\right)}{\sum_{j=1}^{n} \exp _{j}\left(\vartheta_{\beta_{j}} / k\right)}} \\
& \Rightarrow 1-\left(1-\prod_{j=1}^{n}\left(1-\left(1-T_{\alpha_{j}}\right)^{\lambda}\right)^{\frac{\exp \left(\vartheta_{\alpha_{j}} / k\right)}{\sum_{j=1}^{n} e^{\exp \left(\vartheta_{\alpha_{j}} / k\right)}}}\right)^{\frac{1}{\lambda}} \leq 1-\left(1-\prod_{j=1}^{n}\left(1-\left(1-T_{\beta_{j}}\right)^{\lambda}\right)^{\frac{\exp \left(\vartheta_{\beta_{j}} / k\right)}{\sum_{j=1}^{n} \operatorname{exp(\vartheta _{\beta _{j}}/k)}}}\right)^{\frac{1}{\lambda}}
\end{aligned}
$$


Further,

$$
\begin{aligned}
& I_{\alpha_{j}} \geq I_{\beta_{j}} \Rightarrow I_{\alpha_{j}}^{\lambda} \geq I_{\beta_{j}}^{\lambda} \Rightarrow\left(1-I_{\alpha_{j}}^{\lambda}\right) \leq\left(1-I_{\beta_{j}}^{\lambda}\right) \\
& \Rightarrow\left(1-I_{\alpha_{j}}^{\lambda}\right)^{\frac{\exp \left(\vartheta_{\alpha_{j}} / k\right)}{\sum_{j=1}^{n} \exp \left(\vartheta_{\alpha_{j}} / k\right)}} \leq\left(1-I_{\beta_{j}}^{\lambda}\right)^{\frac{\exp \left(\vartheta_{\beta_{j}} / k\right)}{\sum_{j=1}^{n} \exp \left(\vartheta_{\beta_{j}} / k\right)}} \\
& \Rightarrow \prod_{j=1}^{n}\left(1-I_{\alpha_{j}}^{\lambda}\right)^{\frac{\exp \left(\vartheta_{\alpha_{j}} / k\right)}{\sum_{j=1}^{n} \exp \left(\vartheta_{\alpha_{j}} / k\right)}} \leq \prod_{j=1}^{n}\left(1-I_{\beta_{j}}^{\lambda}\right)^{\frac{\exp \left(\vartheta_{\beta_{j}} / k\right)}{\sum_{j=1}^{n} \exp \left(\vartheta_{\beta_{j}} / k\right)}} \\
& \Rightarrow\left(1-\prod_{j=1}^{n}\left(1-I_{\alpha_{j}}^{\lambda}\right)^{\frac{\exp \left(\vartheta_{\alpha_{j}} / k\right)}{\sum_{j=1}^{n} \exp \left(\vartheta_{\alpha_{j}} / k\right)}}\right) \geq\left(1-\prod_{j=1}^{n}\left(1-I_{\beta_{j}}^{\lambda}\right)^{\frac{\exp \left(\vartheta_{\beta_{j}} / k\right)}{\sum_{j=1}^{n} \exp \left(\vartheta_{\beta_{j}} / k\right)}}\right) \\
& \Rightarrow\left(1-\prod_{j=1}^{n}\left(1-I_{\alpha_{j}}^{\lambda}\right)^{\frac{\exp \left(\vartheta_{\alpha_{j}} / k\right)}{\sum_{j=1}^{n} \exp _{\left(\vartheta_{\alpha_{j}} / k\right)}}}\right)^{\frac{1}{\lambda}} \geq\left(1-\prod_{j=1}^{n}\left(1-I_{\beta_{j}}^{\lambda}\right)^{\frac{\exp \left(\vartheta_{\beta_{j}} / k\right)}{\sum_{j=1}^{n} \exp _{\left(\vartheta_{\beta_{j}} / k\right)}}}\right)^{\frac{1}{\lambda}}
\end{aligned}
$$

Similarly, we can also show that

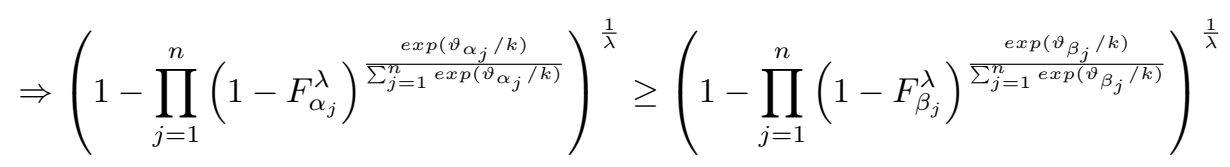

Hence the proof is completed.

\section{Property 3: Boundedness}

Let $\alpha_{j}(j=1,2, \ldots, n)$ be any set of SVN-numbers. If $\alpha^{-}=\min \left\{\alpha_{j}\right\}$ and $\alpha^{+}=\max \left\{\alpha_{j}\right\}$, then $\alpha^{-} \leq \operatorname{GSSVNWG}\left(\alpha_{1}, \alpha_{2}, \ldots, \alpha_{n}\right) \leq \alpha^{+}$.

Proof : Let $\alpha^{+}=\max \left\{\alpha_{1}, \alpha_{2}, \ldots, \alpha_{n}\right\}$ and $\alpha^{-}=\min \left\{\alpha_{1}, \alpha_{2}, \ldots, \alpha_{n}\right\}$.

According to the Property 1 and 2, we have $G S S V N W G\left(\alpha_{1}, \alpha_{2}, \ldots, \alpha_{n}\right) \geq G S S V N W G\left(\alpha^{-}, \alpha^{-}, \ldots, \alpha^{-}\right)=\alpha^{-}$

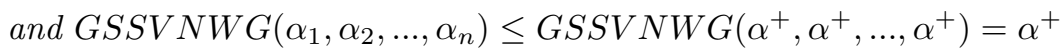

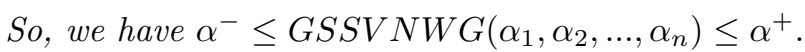

Hence the proof is completed.

\section{MADM under SVN environment based on proposed operators}

The main goal of a MADM strategy is to find the one or more alternative which satisfies the objective of decision maker from a set of possible alternatives w.r.t significant attributes. Using the proposed aggregation operators a MADM strategy under SVN environment is considered. A MADM strategy is presented here ton show the application of proposed approach.

4.1 Decision making approach based on proposed operators

Let $\Psi=\left\{\psi_{1}, \psi_{2}, \ldots, \psi_{m}\right\}$ and $C=\left\{C_{1}, C_{2}, \ldots, C_{n}\right\}$ be the possible set of alternatives and attributes respectively. Let $W=\left\{w_{1}, w_{2}, \ldots, w_{n}\right\}$ be the weight vector of attributes $C_{j}(\mathrm{j}=1,2,3,, \mathrm{n})$, where $w_{j} \geq 0$ and $\sum_{j=1}^{n} w_{j}=1$. Now, we have described the steps of proposed MADM strategy by following algorithm.

\section{Algorithm:}

Step 1: Formulate the decision matrix 
For MADM with SVNnumber information, the rating values of the alternative $\psi_{i}(i=1,2, \ldots, m)$ on the basis of attribute $C_{j}(j=1,2, \ldots, n)$ can be expressed in SVNnumber as $a_{i j}$ where $(\mathrm{i}=1,2,3,, \mathrm{~m} ; \mathrm{j}=1,2,3,, \mathrm{n})$. The decision matrix is represented as follows:

$$
\left[A_{i j}\right]_{m \times n}=\begin{aligned}
& \psi_{1} \\
& \psi_{2} \\
& \vdots \\
& \psi_{m}
\end{aligned}\left(\begin{array}{cccc}
a_{11} & a_{12} & \cdots & a_{1 n} \\
a_{21} & a_{22} & \cdots & a_{2 n} \\
\vdots & \vdots & \ddots & \vdots \\
a_{m 1} & a_{m 2} & \cdots & a_{m n}
\end{array}\right)
$$

is called an decision making matrix.

Step 2: Compute the score matrix and $\vartheta_{i j}$ value matrix

Using Eq. (1) and Eq. (2), We calculate the score value of each alternative for different attribute and represent as matrix form as:

$$
\left[S_{i j}\right]_{m \times n}=\begin{aligned}
& \psi_{1} \\
& \psi_{2} \\
& \vdots \\
& \psi_{m}
\end{aligned}\left(\begin{array}{cccc}
C_{11} & C_{2} & \cdots & C_{n} \\
s_{21} & s_{22} & \cdots & s_{1 n} \\
\vdots & \vdots & \ddots & \vdots \\
s_{m 1} & s_{m 2} & \cdots & s_{m n}
\end{array}\right)
$$

and calculate $\vartheta_{i j}$ value using Eq. (3), represent as follows:

$$
\left[\vartheta_{i j}\right]_{m \times n}=\begin{aligned}
& C_{1} \\
& \psi_{1} \\
& \psi_{2} \\
& \vdots \\
& \psi_{m}
\end{aligned}\left(\begin{array}{cccc}
\vartheta_{11} & \vartheta_{12} & \cdots & \vartheta_{1 n} \\
\vartheta_{21} & \vartheta_{22} & \cdots & \vartheta_{2 n} \\
\vdots & \vdots & \ddots & \vdots \\
\vartheta_{m 1} & \vartheta_{m 2} & \cdots & \vartheta_{m n}
\end{array}\right)
$$

Step 3: Compute weighted matrix

We calculate weight of each alterntive for each attribute by the Eq. (3) and represent in matrix form as:

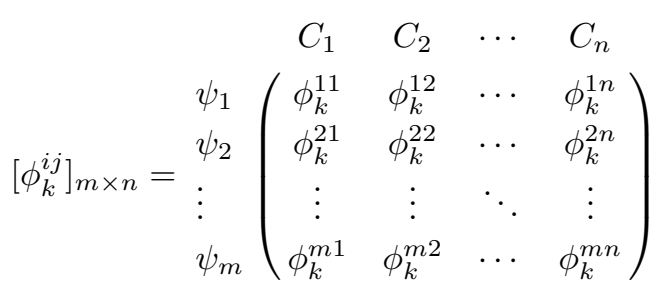

Where, $\phi_{k}^{i j}=\frac{\exp \left(\vartheta_{i j} / k\right)}{\sum_{j=1}^{n} \exp \left(\vartheta_{i j} / k\right)}, \vartheta_{i j}=\left\{\begin{array}{ll}\prod_{i=1}^{j-1} S_{i}, \mathrm{j}=2,3, \ldots, n \\ 1 \quad \mathrm{j}=1\end{array}, S_{i}\right.$ is the score function of the SVNN $\alpha_{i}$.

Step 4: Aggregate the all attributes

Using aggregation operators we aggragate the all attribute values for respective alternative and results are shown in table form as:

Step 5: Ranking the alternatives

Based on the score value (From Table 1) of alternative, we arranged the ranking odrer of alternatives using Eq. (1) and Eq. (2).

Step 6: End the algorithm. 
Table 1 The aggregated SVNNs and score values of aggregated SVNNs

\begin{tabular}{ccc}
\hline Alternatives & Aggregated SVNNs & Score values \\
\hline$\psi_{1}$ & $\tilde{a_{1}}$ & $\tilde{S_{1}}$ \\
$\psi_{2}$ & $\tilde{a_{2}}$ & $\tilde{S_{2}}$ \\
$\cdot$ & $\cdot$ & $\cdot$ \\
$\cdot$ & $\cdot$ & $\cdot$ \\
$\psi_{m}$ & $\tilde{a_{m}}$ & $\tilde{S_{m}}$ \\
\hline
\end{tabular}

\section{Numerical illustration}

In every year worldwide, many peoples are affected by various natural disasters. These types of disaster are Hurricanes and Tropical Storms, Drought, Wildfires, Floods, Earthquakes, Tornadoes and Severe Storms, etc. The most and common thoughtful nature disaster is Flood disaster. Flood disaster problem can handle by MADM strategy according to given information Yu [17]. In Flood disaster control and mitigation, risk decision and evaluation are very significant steps. According our knowledge [21] we have composed four important attributes to evaluate the risk of Flood disaster, which are:

i) Disaster-inducing factors $\left(C_{1}\right)$,

ii) Hazard-formative environment $\left(C_{2}\right)$,

iii) Characters of hazard affected body $\left(C_{3}\right)$, and

iv) Social disaster bearing capacity $\left(C_{4}\right)$.

Apparently, these evaluation attribute are complicated and difficult to characterize quantitatively. We can handle this type of difficulties considering the attributes information by SVN set.

Let us assume that $\psi_{1}, \psi_{2}, \psi_{3}$, and $\psi_{4}$ are the four maritime cities in India. Our aim is to find the best city according to the four attributes. We expressed the appraisement informations of four cities according to the four attributes in terms of SVN-set. Now, we will solved this decision making problem using the proposed operators. Step 1: Formulate the decision matrix

The appraisement informations of four cities consider by SVN-number according to the four attributes. Representation of the decision matrix shown in Eq. (16) as given by:

$$
\left.\begin{array}{ccccc}
C_{1} & C_{2} & C_{3} & C_{4} \\
{[A]_{4 \times 4}=} & \psi_{1} & \psi_{2} \\
\psi_{3} & \langle 0.6,0.4,0.3\rangle & \langle 0.7,0.3,0.4\rangle & \langle 0.8,0.4,0.6\rangle & \langle 0.6,0.2,0.4\rangle \\
\psi_{4} & \langle 0.3,0.1,0.4\rangle & \langle 0.5,0.2,0.2\rangle & \langle 0.8,0.3,0.4\rangle & \langle 0.6,0.3,0.5\rangle \\
\langle 0.6,0.3,0.5\rangle & \langle 0.7,0.3,0.5\rangle & \langle 0.8,0.3,0.5\rangle & \langle 0.4,0.3,0.2\rangle \\
\langle 0.7,0.3,0.3\rangle & \langle 0.3,0.4,0.3\rangle & \langle 0.7,0.4,0.5\rangle & \langle 0.8,0.3,0.4\rangle
\end{array}\right)
$$

Step 2: Compute the score matrix and $\vartheta_{i j}$ value matrix

Using Eq. (1), We calculate the score value of each alternative for different attribute and represent as matrix form as:

$$
[S]_{4 \times 4}=\begin{array}{cccc}
C_{1} & C_{2} & C_{3} & C_{4} \\
\psi_{1} \\
\psi_{2} \\
\psi_{3} \\
\psi_{4}
\end{array}\left(\begin{array}{cccc}
0.63 & 0.67 & 0.60 & 0.67 \\
0.60 & 0.70 & 0.70 & 0.60 \\
0.60 & 0.63 & 0.67 & 0.63 \\
0.70 & 0.53 & 0.60 & 0.70
\end{array}\right)
$$


and calculate $\vartheta_{i j}$ value using Eq. (4), represent as follows:

$$
\begin{gathered}
C_{1} \\
{[\vartheta]_{4 \times 4}=} \\
\psi_{1} \\
\psi_{2} \\
\psi_{3} \\
\psi_{4}
\end{gathered}\left(\begin{array}{cccc}
1 & 0.63 & 0.42 & 0.28 \\
1 & 0.60 & 0.42 & 0.29 \\
1 & 0.60 & 0.38 & 0.25 \\
1 & 0.70 & 0.37 & 0.22
\end{array}\right)
$$

Step 3: Compute $\phi_{k}^{i j}$ matrix (Let parameter $k=1$ )

We calculate the values of $\phi_{k}^{i j}$ for each alternative with respects to each attributes by the Eq. (3) and represent in matrix form as:

$$
\begin{aligned}
& \begin{array}{llll}
C_{1} & C_{2} & C_{3} & C_{4}
\end{array} \\
& {[\phi]_{4 \times 4}=\begin{array}{c}
\psi_{1} \\
\psi_{2} \\
\psi_{3} \\
\psi_{4}
\end{array}\left(\begin{array}{cccc}
0.36 & 0.25 & 0.20 & 0.18 \\
0.34 & 0.24 & 0.21 & 0.18 \\
0.37 & 0.25 & 0.20 & 0.18 \\
0.37 & 0.27 & 0.20 & 0.17
\end{array}\right)}
\end{aligned}
$$

Step 4: Aggregate the all attribute values of alternatives

Based on the SSVNWA operator, the aggregated SVN-numbers and score values of Eq. (17) are shown in Table 2 (Parameter $k=1$ fixed).

From Table 2, we find the riskiest city is $\psi_{2}$.

Based on the SSVNWG operator, the aggregated SVN-numbers and corresponding score values of Eq. (16) are shown in Table 3 (Parameter $k=1$ fixed),

From Table 3, we find the riskiest city is $\psi_{2}$.

Based on the GSSVNWA operator, the aggregated SVN-numbers and corresponding score values of Eq. (16) are shown in Table 4(Parameter $\lambda=2,5,10$ and Parameter $k=1$ fixed).

From Table 4, we find the riskiest city is $\psi_{4}$ for $\lambda=2$ and $\psi_{2}$ for $\lambda=5,10$.

Based on the GSSVNWG operator, the aggregated SVN-numbers and corresponding score values of Eq. (16) are shown in Table 5 (Parameter $\lambda=2,5,10$ and Parameter $k=1$ fixed).

From Table 5, we find the riskiest city is $\psi_{1}$ for $\lambda=2,5,10$.

Table 2 The aggregated SVN-numbers and score values based on SSVNWA operator

\begin{tabular}{ccc}
\hline Alternatives & Aggregated SVNNs & Score values \\
\hline$\psi_{1}$ & $\langle 0.622,0.332,0.398\rangle$ & 0.631 \\
$\psi_{2}$ & $\langle 0.546,0.194,0.362\rangle$ & $\mathbf{0 . 6 6 3}$ \\
$\psi_{3}$ & $\langle 0.651,0.300,0.424\rangle$ & 0.642 \\
$\psi_{4}$ & $\langle 0.652,0.339,0.345\rangle$ & 0.656 \\
\hline
\end{tabular}

Step 5: Ranking order of alternatives

According to the decreasing score value of alternatives $\psi_{i}(i=1,2,3,4)$ and based on the Table 2, Table 3, Table 4 and Table 5 , the ranking order of alternatives is presented in Table 6 .

Step 6: The procedure of proposed algorithm end here.

In the the numerical example we analysed FD-risk assessment problem. It easy to recognize that the neutrosophic set of information is expressed by SVN-number. Here we have been examined in details for the FD-risk 
Table 3 The aggregated SVNNs and score values based on SSVNWG operator

\begin{tabular}{ccc}
\hline Alternatives & Aggregated SVNNs & Score values \\
\hline$\psi_{1}$ & $\langle 0.633,0.340,0.414\rangle$ & 0.636 \\
$\psi_{2}$ & $\langle 0.489,0.204,0.368\rangle$ & $\mathbf{0 . 6 6 9}$ \\
$\psi_{3}$ & $\langle 0.614,0.300,0.456\rangle$ & 0.619 \\
$\psi_{4}$ & $\langle 0.568,0.351,0.365\rangle$ & 0.617 \\
\hline
\end{tabular}

Table 4 The aggregated SVNNs and score values based on GSSVNWA operator

\begin{tabular}{cccc}
\hline value of $\lambda$ & Alternatives & Aggregated SVNNs & Score values \\
\hline \multirow{4}{*}{$\lambda=2$} & $\psi_{1}$ & $\langle 0.679,0.327,0.388\rangle$ & 0.655 \\
& $\psi_{2}$ & $\langle 0.572,0.189,0.353\rangle$ & 0.677 \\
& $\psi_{3}$ & $\langle 0.659,0.300,0.414\rangle$ & 0.648 \\
& $\psi_{4}$ & $\langle 0.665,0.245,0.343\rangle$ & $\mathbf{0 . 6 9 2}$ \\
$\lambda=5$ & & & \\
& $\psi_{1}$ & $\langle 0.689,0.316,0.372\rangle$ & 0.667 \\
& $\psi_{2}$ & $\langle 0.632,0.178,0.330\rangle$ & $\mathbf{0 . 7 0 8}$ \\
& $\psi_{3}$ & $\langle 0.680,0.300,0.378\rangle$ & 0.643 \\
& $\psi_{4}$ & $\langle 0.692,0.337,0.336\rangle$ & 0.673 \\
& & & \\
& $\psi_{1}$ & $\langle 0.710,0.294,0.353\rangle$ & 0.688 \\
& $\psi_{2}$ & $\langle 0.691,0.163,0.295\rangle$ & $\mathbf{0 . 7 4 4}$ \\
& $\psi_{3}$ & $\langle 0.708,0.300,0.320\rangle$ & 0.696 \\
& $\psi_{4}$ & $\langle 0.715,0.330,0.326\rangle$ & 0.686 \\
\hline
\end{tabular}

Table 5 The aggregated SVNNs and score values based on GSSVNWG operator

\begin{tabular}{cccc}
\hline value of $\lambda$ & Alternatives & Aggregated SVNNs & Score values \\
\hline \multirow{4}{*}{$\lambda=2$} & $\psi_{1}$ & $\langle 0.656,0.347,0.424\rangle$ & $\mathbf{0 . 6 2 8}$ \\
& $\psi_{2}$ & $\langle 0.464,0.221,0.382\rangle$ & 0.620 \\
& $\psi_{3}$ & $\langle 0.598,0.300,0.465\rangle$ & 0.611 \\
& $\psi_{4}$ & $\langle 0.536,0.353,0.370\rangle$ & 0.604 \\
& & & \\
& $\psi_{1}$ & $\langle 0.638,0.364,0.463\rangle$ & $\mathbf{0 . 6 0 3}$ \\
& $\psi_{2}$ & $\langle 0.413,0.253,0.408\rangle$ & 0.584 \\
& $\psi_{3}$ & $\langle 0.548,0.300,0.481\rangle$ & 0.589 \\
& $\psi_{4}$ & $\langle 0.451,0.361,0.395\rangle$ & 0.565 \\
& & & \\
& $\psi_{1}$ & $\langle 0.623,0.378,0.513\rangle$ & $\mathbf{0 . 5 7 7}$ \\
& $\psi_{2}$ & $\langle 0.369,0.273,0.433\rangle$ & 0.554 \\
& $\psi_{3}$ & $\langle 0.492,0.300,0.490\rangle$ & 0.567 \\
& $\psi_{4}$ & $\langle 0.385,0.373,0.430\rangle$ & 0.527
\end{tabular}

assessment problem. Also the process of proposed strategies are reasonable for this problem. From the numerical example, we can say that it is comfortable to use the strategy to cope with the other risk assessment problems. Therefore the proposed decision making strategy has a deep practical value. 
Table 6 Ranking order of alternatives and riskiest city for various operators

\begin{tabular}{|c|c|c|}
\hline Proposed operators & Ranking order of alternatives & riskiest city \\
\hline$S S V N W A$ & $\psi_{2} \succ \psi_{4} \succ \psi_{3} \succ \psi_{1}$ & $\psi_{2}$ \\
\hline$S S V N W G$ & $\psi_{2} \succ \psi_{1} \succ \psi_{3} \succ \psi_{4}$ & $\psi_{2}$ \\
\hline$G S S V N W A, \lambda=2$ & $\psi_{4} \succ \psi_{2} \succ \psi_{1} \succ \psi_{3}$ & $\psi_{4}$ \\
\hline$\lambda=5$ & $\psi_{2} \succ \psi_{4} \succ \psi_{1} \succ \psi_{3}$ & $\psi_{2}$ \\
\hline$\lambda=10$ & $\psi_{2} \succ \psi_{3} \succ \psi_{1} \succ \psi_{4}$ & $\psi_{2}$ \\
\hline$G S S V N W G, \lambda=2$ & $\psi_{1} \succ \psi_{2} \succ \psi_{3} \succ \psi_{4}$ & $\psi_{1}$ \\
\hline$\lambda=5$ & $\psi_{1} \succ \psi_{3} \succ \psi_{2} \succ \psi_{4}$ & $\psi_{1}$ \\
\hline$\lambda=10$ & $\psi_{1} \succ \psi_{3} \succ \psi_{2} \succ \psi_{4}$ & $\psi_{1}$ \\
\hline
\end{tabular}

\section{Conclusions}

In recent years, aggregation operator have become a popular research topic in the fields of decision making. In this paper, we have presented some new aggregation operators for solving an real MADM problem under SVNenvironment. Additionally, some different aggregation operators are developed, which are softmax SVN weighted average (SVNWA) operator, softmax SVN weighted geometric (SVNWG) operator, generalized softmax SVN weighted average (GSSVNWA) operator and generalized softmax SVN weighted geometric (GSSVNIFWG) operator. Then, we have presented the some essential properties of these operators. Moreover, using the proposed operators, we have been built a MADM strategy under SVN-environment. Finally, we illustrated one example numerically to express the usefulness and effectiveness of the proposed MADM technique.

In the future, we will extend the proposed operators in interval neutrosophic set [22], neutrosophic cubic set [23], refined neutrosophic set [24] environments. Also, we will try to apply the proposed operators in different realistic decision making problem.

\section{Compliance with ethical standards}

Conflict of interest: NA.

Ethical approval: NA.

Funding: Not applicable (NA)

Authors Contribution All author's contributed equally.

\section{References}

1. Wang H, Smarandache F, Zhang YQ, Sunderraman R (2010) Single valued neutrosophic sets, Multispace multi-structure, 4:410413.

2. Zadeh LA (1965) Fuzzy sets, Information Control, 8:338-356.

3. Atanassov K (1986) Intuitionistic fuzzy sets, Fuzzy Sets \& Systems, 20:87-96.

4. Jun Ye (2013) Multi-criteria decision-making method using the correlation coefficient under single-valued neutrosophic environment, International Journal of General Systems 4:386-394.

5. Sodenkamp MA, Tavana M, Caprio DD (2018) An aggregation method for solving group multi-criteria decision-making problems with single-valued neutrosophic sets, Applied Soft Computing, 71:715-727.

6. Wei G, Wei Y (2018) Some single-valued neutrosophic dombi prioritized weighted aggregation operators in multiple attribute decision making, Journal of Intelligent \& Fuzzy Systems, 25:1-13.

7. Ren, S (2017) Multi-criteria decision-making method under a single valued neutrosophic environment, International Journal of Intelligent Information Technologies, 13:23-37.

8. Biswas P, Pramanik S, Giri BC (2016) TOPSIS method for multi-attribute group decision-making under single-valued neutrosophic environment, Neural Computing and Applications, 27:727-737.

9. Pramanik S, Biswas P, Giri BC (2017) Hybrid vector similarity measures and their applications to multi-attribute decision making under neutrosophic environment, Neural computing and Applications, 28:1163-1176. 
10. Garg H, Nancy (2018) Some hybrid weighted aggregation operator sunder neutrosophic set environment and their applications to multi-criteria decision-making, Application Intelligent, 48: 48-71.

11. Ji P, Wang JQ, Zhang HY, (2016) Frank prioritized bonferroni mean operator with single-valued neutrosophic sets and its application in selecting third-party logistics providers. Neural Computing and Applications, 14:1-25.

12. Nancy, Garg H (2016) Novel single-valued neutrosophic decision making operators under frank norm operations and its application. International Journal of Uncertain Quantify, 6:361375.

13. Liu P, Chu Y, Li Y, Chen, Y (2014) Some Generalized neutrosophic number hamacher aggregation operators and their application to group decision making, International Journal of Fuzzy Systems, 16:1-22.

14. Chen J, Ye J (2017). Some single-valued neutrosophic dombi weighted aggregation operators for multiple attribute decisionmaking Symmetry, 9:82.

15. Li Y, Liu P, Chen Y, (2016) Some single valued neutrosophic number heronian mean operators and their application in multiple attribute group decision making, Informatica, 27:85-110.

16. Torres R, Salas R, Astudillo H (2014) Time-based hesitant fuzzy information aggregation approach for decision making problems. International Journal Intelligent Systems, 29:579-595.

17. Yu D (2016) Softmax function based intuitionistic fuzzy multi-criteria decision making and applications, Operational Research, $16: 327-348$.

18. Zhang HY, Wang JQ, Chen XH (2014) Interval neutrosophic sets and their application in multi-criteria decision making problems, The Scientific World Journal, 2014:645953.

19. Jacobs RA, Jordan MI, Nowlan SJ, Hinton GE (1991) Adaptive mixtures of local experts. Neural Computing, 3:79-87.

20. Torres R, Salas R, Allende H, Moraga C (2003) Robust expectation maximization learning algorithm for mixture of experts, International Computational Methods in Neural Modelling:7th international work conference on artificial and natural neural networks, Springer, Berlin, Heidelberg, New York, 2003:238-245.

21. Yang XL (2012) Research on the application of MADM in flood risk evaluation. Doctoral dissertation, Huazhong University of Science and Technology.

22. Wang H, Smarandache F, Zhang YQ, Sunderraman R (2005) Interval neutrosophic sets and logic: Theory and applications in computing, Hexis, Phoenix, AZ.

23. Ali M, Deli I, Smarandache F (2016) The theory of neutrosophic cubic sets and their applications in pattern recognition. Journal of Intelligent Fuzzy Systems, 30:1957-1963.

24. Smarandache F, N-Valued refined neutrosophic logic and its applications in physics, Prog. Phys., 4:143-146.

25. Smarandache F (2005) A unifying field in logics: neutrosophic logic. Neutrosophy, neutrosophic probability and statistics. American Research Press, Rehoboth.

26. Garai T, Garg H, Roy TK (2020)A ranking method based on possibility mean for multi-attribute decision making with single valued neutrosophic numbers. Journal of Ambient Intelligence and Humanized Computing, 11:1-14.

27. Garai T, Dalapati S, Garg H, Roy TK (2020) Possibility mean, variance and standard deviation of single-valued neutrosophic numbers and its applications to multi-attribute decision-making problems. Soft Computing, 24:18795-18809.

28. Liu P, Khan Q, Mahmood T (2019) Multiple-attribute decision making based on single-valued neutrosophic schweizer-sklar prioritized aggregation operator. Cognitive Systems Research, 57,:175-196.

29. Liu P, Khan Q, Mahmood T (2019) Some single-valued neutrosophic power muirhead mean operators and their application to group decision making. Journal of Intelligent \& Fuzzy Systems, 37:2515-2537.

30. Tan, RP, Zhang W (2021) Decision-making method based on new entropy and refined single-valued neutrosophic sets and its application in typhoon disaster assessment. Applied Intelligence, 51:283-307.

31. Rong Y, Liu Y, Pei Z (2020) Generalized single-valued neutrosophic power aggregation operators based on archimedean copula and Co-copula and their application to multi-attribute decision-making, IEEE Access, 8:35496-35519. 